\title{
Determinization and Limit-determinization of Emerson-Lei automata
}

\author{
Tobias John ${ }^{[0000-0001-5855-6632]}$, Simon Jantsch ${ }^{[0000-0003-1692-2408]}$, \\ Christel Baier ${ }^{[0000-0002-5321-9343]}$, and Sascha Klüppelholz ${ }^{[0000-0003-1724-2586]}$ \\ Technische Universität Dresden * \\ tobiasj@posteo.de \\ $\{$ simon.jantsch, christel.baier, sascha.klueppelholz\}@tu-dresden.de
}

\begin{abstract}
We study the problem of determinizing $\omega$-automata whose acceptance condition is defined on the transitions using Boolean formulas, also known as transition-based Emerson-Lei automata (TELA). The standard approach to determinize TELA first constructs an equivalent generalized Büchi automaton (GBA), which is later determinized. We introduce three new ways of translating TELA to GBA. Furthermore, we give a new determinization construction which determinizes several GBA separately and combines them using a product construction. An experimental evaluation shows that the product approach is competitive when compared with state-of-the-art determinization procedures. We also study limit-determinization of TELA and show that this can be done with a single-exponential blow-up, in contrast to the known doubleexponential lower-bound for determinization. Finally, one version of the limit-determinization procedure yields good-for-MDP automata which can be used for quantitative probabilistic model checking.
\end{abstract}

\section{Introduction}

Automata on infinite words, also called $\omega$-automata, play a fundamental role in the fields of verification and synthesis of reactive systems $12,31,34,38$. They can be used both to represent properties of systems and the systems themselves. For logical specification languages such as linear temporal logic (LTL), many verification systems, such as SPIN [5] or PRISM 24], use logic-toautomata translations internally to verify a given system against the specification.

A major research question in this area has been, and still is, the question of whether and how $\omega$-automata can be determinized efficiently $25,30,32,35,36$. The first single-exponential and asymptotically optimal determinization for Büchi automata was presented in 35]. Deterministic automata are important from a practical point of view as classical automata-based solutions to reactive synthesis and probabilistic verification use deterministic automata 31, 38.

\footnotetext{
* This work was funded by DFG grant 389792660 as part of TRR 248, the Cluster of Excellence EXC 2050/1 (CeTI, project ID 390696704, as part of Germany's Excellence Strategy), DFG-projects BA-1679/11-1 and BA-1679/12-1, and the Research Training Group QuantLA (GRK 1763).
} 
The high complexity of determinization and most logic-to-automata translations have raised the question of more succinct representations of $\omega$-automata. Using generalized acceptance conditions (e.g. generalized Büchi 13 or generalized Rabin [9, 11]) and transition-based [18, rather than state-based, conditions are common techniques in this direction. An even more general approach has led to the HOA-format 2], which represents the acceptance condition as a positive Boolean formula over standard Büchi (Inf) and co-Büchi (Fin) conditions, also called Emerson-Lei conditions [1, 34]. Together with a vast body of work on heuristics and dedicated procedures this standardized format has led to practically usable and mature tools and libraries such as SPOT [15] and OwL [23] which support a wide range of operations on $\omega$-automata. Special classes of nondeterministic automata with some of the desired properties of deterministic automata have also been studied. The classes of good-for-MDP [20] and good-for-games 22 automata can be used for quantitative probabilistic model checking of Markov decision processes 19, 37, while limit-deterministic Büchi automata can be used for qualitative model-checking 12 . Dedicated translations from LTL directly to deterministic and limit-deterministic automata have been considered in 17 .

This paper considers determinization and limit-determinization of TELA. In contrast to limit-determinization, the theoretical complexity of determinization is well understood (a tight, double-exponential, bound was given in [34, 35]). However, it has not been studied yet from a practical point of view.

Contribution. We propose three new translations from TELA to GBA (Sec. 3) and give an example in which they perform exponentially better than state-of-theart implementations. We introduce a new determinization procedure for TELA based on a product construction (Sec. 4). Our experiments show that it often outperforms the approaches based on determinizing a single GBA (Sec. 6). A simple adaptation of the product construction produces limit-deterministic TELA of single-exponential size (in contrast to the double-exponential worst-case complexity of full determinization, Sec. 5.1). We show that deciding $\operatorname{Pr}_{\mathcal{M}}^{\max }(\mathcal{L}(\mathcal{A}))>0$ is NP-complete for limit-deterministic TELA $\mathcal{A}$, and in $\mathrm{P}$ if the acceptance of $\mathcal{A}$ is fin-less (Prop. 5.9). Finally, we show how to limit-determinize TELA based on the breakpoint-construction. A version of this procedure yields good-for-MDP Büchi automata (Def. 5.6). Thereby $\operatorname{Pr}_{\mathcal{M}}^{\max }(\mathcal{L}(\mathcal{A}))$ is computable in single-exponential time for arbitrary MDP $\mathcal{M}$ and TELA $\mathcal{A}$ (Thm. 5.15).

Related work. The upper-bound for TELA-determinization 34, 35 relies on a translation to GBA which first transforms the acceptance formula into disjunctive normal form (DNF). We build on this idea. Another way of translating TELA to GBA was described in [14. Translations from LTL to TELA have been proposed in $8,26,29$, and all of them use product constructions to combine automata for subformulas. The emptiness-check for $\omega$-automata under different types of acceptance conditions has been studied in 3, 9, 11, 16, where 3 covers the general case of Emerson-Lei conditions and also considers qualitative probabilistic model checking using deterministic TELA. The generalized Rabin condition from 9, 11 is equivalent to the special DNF that we use and a special case of the hyper-Rabin condition for which the emptiness problem is in $\mathrm{P}$ [1, 10. Probabilistic model 
checking for deterministic automata under this condition is considered in 11, while 9 is concerned with standard emptiness while allowing nondeterminism. A procedure to transform TELA into parity automata is presented in 33 .

\section{Preliminaries}

Automata. A transition-based Emerson-Lei automaton (TELA) $\mathcal{A}$ is a tuple $(Q, \Sigma, \delta, I, \alpha)$, where $Q$ is a finite set of states, $\Sigma$ is a finite alphabet, $\delta \subseteq Q \times \Sigma \times Q$ is the transition relation, $I \subseteq Q$ is the set of initial states and $\alpha$ is a symbolic acceptance condition over $\delta$, which is defined by:

$$
\alpha::=t|f f| \operatorname{Inf}(T)|\operatorname{Fin}(T)|(\alpha \vee \alpha) \mid(\alpha \wedge \alpha) \text {, with } T \subseteq \delta
$$

If $\alpha$ is $t, f f, \operatorname{Inf}(T)$ or $\operatorname{Fin}(T)$, then it is called atomic. We denote by $|\alpha|$ the number of atomic conditions contained in $\alpha$, where multiple occurrences of the same atomic condition are counted multiple times. Symbolic acceptance conditions describe sets of transitions $T \subseteq \delta$. Their semantics is defined recursively as follows:

$$
T \models \# \quad T \models \operatorname{Inf}\left(T^{\prime}\right) \text { iff } T \cap T^{\prime} \neq \emptyset \quad T \models \alpha_{1} \vee \alpha_{2} \text { iff } T \models \alpha_{1} \text { or } T \models \alpha_{2}
$$$$
T \not \models \text { ff } \quad T \models \operatorname{Fin}\left(T^{\prime}\right) \text { iff } T \cap T^{\prime}=\emptyset \quad T \models \alpha_{1} \wedge \alpha_{2} \text { iff } T \models \alpha_{1} \text { and } T \models \alpha_{2}
$$

Two acceptance conditions $\alpha$ and $\beta$ are $\delta$-equivalent $\left(\alpha \equiv_{\delta} \beta\right)$ if for all $T \subseteq \delta$ we have $T \models \alpha \Longleftrightarrow T \models \beta$. A run of $\mathcal{A}$ for an infinite word $u=u_{0} u_{1} \ldots \in \Sigma^{\omega}$ is an infinite sequence of transitions $\rho=\left(q_{0}, u_{0}, q_{1}\right)\left(q_{1}, u_{1}, q_{2}\right) \ldots \in \delta^{\omega}$ that starts with an initial state $q_{0} \in I$. The set of transitions that appear infinitely often in $\rho$ are denoted by $\inf (\rho)$. A run $\rho$ is accepting $(\rho \models \alpha)$ iff $\inf (\rho) \models \alpha$. The language of $\mathcal{A}$, denoted by $\mathcal{L}(\mathcal{A})$, is the set of all words for which there exists an accepting run of $\mathcal{A}$. The sets of infinite words which are the language of some TELA are called $\omega$-regular. A TELA $\mathcal{A}$ is deterministic if the set of initial states contains exactly one state and the transition relation is a function $\delta: Q \times \Sigma \rightarrow Q$. It is complete, if for all $(q, a) \in Q \times \Sigma: \delta \cap\left\{\left(q, a, q^{\prime}\right) \mid q^{\prime} \in Q\right\} \neq \varnothing$. A Büchi condition is an acceptance condition of the form $\operatorname{Inf}(T)$ and a generalized Büchi condition is a condition of the form $\bigwedge_{1 \leq i \leq k} \operatorname{Inf}\left(T_{i}\right)$. We call the sets $T_{i}$ appearing in a generalized Büchi condition its acceptance sets. Rabin (resp. Street) conditions are of the form $\bigvee_{1 \leq i \leq k}\left(\operatorname{Fin}\left(F_{i}\right) \wedge \operatorname{Inf}\left(T_{i}\right)\right)\left(\operatorname{resp} . \bigwedge_{1 \leq i \leq k}\left(\operatorname{Fin}\left(F_{i}\right) \vee \operatorname{Inf}\left(T_{i}\right)\right)\right)$.

Probabilistic systems. A labeled Markov decision process (MDP) $\mathcal{M}$ is a tuple $\left(S, s_{0}\right.$, Act, $\left.P, \Sigma, L\right)$ where $S$ is a finite set of states, $s_{0} \in S$ is the initial state, Act is a finite set of actions, $P: S \times$ Act $\times S \rightarrow[0,1]$ defines the transition probabilities with $\sum_{s^{\prime} \in S} P\left(s, \alpha, s^{\prime}\right) \in\{0,1\}$ for all $(s, \alpha) \in S \times$ Act and $L: S \rightarrow \Sigma$ is a labeling function of the states into a finite alphabet $\Sigma$. Action $\alpha \in$ Act is enabled in $s$ if $\sum_{s^{\prime} \in S} P\left(s, \alpha, s^{\prime}\right)=1$, and $\operatorname{Act}(s)=\{\alpha \mid \alpha$ is enabled in $s\}$. A path of $\mathcal{M}$ is an infinite sequence $s_{0} \alpha_{0} s_{1} \alpha_{1} \ldots \in(S \times \text { Act })^{\omega}$ such that $P\left(s_{i}, \alpha_{i}, s_{i+1}\right)>0$ for all $i \geq 0$. The set of all paths of $\mathcal{M}$ is denoted by $\operatorname{Paths}(\mathcal{M})$ and $\operatorname{Paths}_{\mathrm{fin}}(\mathcal{M})$ denotes the finite paths. Given a path $\pi=s_{0} \alpha_{0} s_{1} \alpha_{1} \ldots$, we let $L(\pi)=L\left(s_{0}\right) L\left(s_{1}\right) \ldots \in$ $\Sigma^{\omega}$. A Markov chain is an MDP with $|\operatorname{Act}(s)| \leq 1$ for all states $s$. A scheduler of $\mathcal{M}$ is a function $\mathfrak{S}:(S \times \text { Act })^{*} \times S \rightarrow$ Act such that $\mathfrak{S}\left(s_{0} \alpha_{0} \ldots s_{n}\right) \in \operatorname{Act}\left(s_{n}\right)$. It induces a Markov chain $\mathcal{M}_{\mathfrak{S}}$ and thereby a probability measure over $\operatorname{Paths}(\mathcal{M})$. 
The probability of a set of paths $\Pi$ starting in $s_{0}$ under this measure is $\operatorname{Pr}_{\mathcal{M}}^{\mathfrak{S}}(\Pi)$. For an $\omega$-regular property $\Phi \subseteq \Sigma^{\omega}$ we define $\operatorname{Pr}_{\mathcal{M}}^{\max }(\Phi)=\sup _{\mathfrak{S}} \operatorname{Pr}_{\mathcal{M}}^{\mathfrak{S}}(\{\pi \mid \pi \epsilon$ Paths $(\mathcal{M})$ and $L(\pi) \in \Phi\})$. See [4, Chapter 10] for more details.

\section{From TELA to Generalized Büchi Automata}

\subsection{Operations on Emerson-Lei Automata}

The first operator takes a TELA and splits it along the top-level disjuncts of the acceptance condition. Let $\mathcal{A}=(Q, \Sigma, \delta, I, \alpha)$ be a TELA where $\alpha=$ $\bigvee_{1<i<m} \alpha_{i}$ and the $\alpha_{i}$ are arbitrary acceptance conditions. We define $\operatorname{split}(\mathcal{A}):=$ $\left(\mathcal{A}_{1}, \ldots, \mathcal{A}_{m}\right)$ with $\mathcal{A}_{i}=\left(Q, \Sigma, \delta, I, \alpha_{i}\right)$ for $1 \leq i \leq m$, and $\operatorname{split}(\mathcal{A})[i]:=\mathcal{A}_{i}$.

Lemma 3.1. It holds that $\mathcal{L}(\mathcal{A})=\bigcup_{1 \leq i \leq m} \mathcal{L}(\operatorname{split}(\mathcal{A})[i])$.

The analogous statement does not hold for conjunction and intersection (cf Fig. 5 in the appendix). We also need constructions to realize the union of a sequence of automata. This can either be done using the sum (i.e. disjoint union) or the disjunctive product of the state spaces. We define a general sum (simply called sum) operation and one that preserves GBA acceptance (called GBA-specific sum). The disjunctive product construction for TELA is mentioned in 14 and similar constructions are used in 26, 29. While the sum operations yield smaller automata in general, only the product construction preserves determinism.

Definition 3.2. Let $\mathcal{A}_{i}=\left(Q_{i}, \Sigma, \delta_{i}, I_{i}, \alpha_{i}\right)$, with $i \in\{0,1\}$, be two complete TELA with disjoint state-spaces. The sum of $\mathcal{A}_{0}$ and $\mathcal{A}_{1}$ is defined as follows:

$$
\mathcal{A}_{0} \oplus \mathcal{A}_{1}=\left(Q_{0} \cup Q_{1}, \Sigma, \delta_{0} \cup \delta_{1}, I_{0} \cup I_{1},\left(\alpha_{0} \wedge \operatorname{Inf}\left(\delta_{0}\right)\right) \vee\left(\alpha_{1} \wedge \operatorname{Inf}\left(\delta_{1}\right)\right)\right.
$$

If $\alpha_{i}=\operatorname{Inf}\left(T_{1}^{i}\right) \wedge \ldots \wedge \operatorname{Inf}\left(T_{k}^{i}\right)$, with $i \in\{0,1\}$, (i.e. both automata are $G B A$ ), then we can use the GBA-specific sum:

$\mathcal{A}_{0} \oplus_{G B A} \mathcal{A}_{1}=\left(Q_{0} \cup Q_{1}, \Sigma, \delta_{0} \cup \delta_{1}, I_{0} \cup I_{1},\left(\operatorname{Inf}\left(T_{1}^{0} \cup T_{1}^{1}\right) \wedge \ldots \wedge \operatorname{Inf}\left(T_{k}^{0} \cup T_{k}^{1}\right)\right)\right)$

The disjunctive product is defined as follows:

$$
\mathcal{A}_{0} \otimes \mathcal{A}_{1}=\left(Q_{0} \times Q_{1}, \Sigma, \delta_{\otimes}, I_{0} \times I_{1},\left(\uparrow\left(\alpha_{0}\right) \vee \uparrow\left(\alpha_{1}\right)\right)\right)
$$

with $\delta_{\otimes}=\left\{\left(\left(q_{0}, q_{1}\right), a,\left(q_{0}^{\prime}, q_{1}^{\prime}\right)\right) \mid\left(q_{0}, a, q_{0}^{\prime}\right) \in \delta_{0}\right.$ and $\left.\left(q_{1}, a, q_{1}^{\prime}\right) \in \delta_{1}\right\}$ and $\uparrow\left(\alpha_{i}\right)$ is constructed by replacing every occurring set of transitions $T$ in $\alpha_{i}$ by $\left\{\left(\left(q_{0}, q_{1}\right), u,\left(q_{0}^{\prime}, q_{1}^{\prime}\right)\right) \in \delta_{\otimes} \mid\left(q_{i}, u, q_{i}^{\prime}\right) \in T\right\}$.

The additional $\operatorname{Inf}\left(\delta_{0}\right)$ and $\operatorname{Inf}\left(\delta_{1}\right)$ atoms in the acceptance condition of $\mathcal{A}_{0} \oplus \mathcal{A}_{1}$ are essential (see Fig. 6 in the appendix). We can apply the GBA-specific sum to any two GBA by adding $\operatorname{Inf}\left(\delta_{i}\right)$ atoms until the acceptance conditions are of equal length. Many of our constructions will require the acceptance condition of the TELA to be in DNF. We will use the following normal form throughout the paper (also called generalized Rabin in 9,11$]$ ).

Definition 3.3 (DNF for TELA). Let $\mathcal{A}=(Q, \Sigma, \delta, I, \alpha)$ be a TELA. We say that $\mathcal{A}$ is in DNF if $\alpha$ is of the form $\alpha=\bigvee_{1 \leq i \leq m} \alpha_{i}$, with $\alpha_{i}=\operatorname{Fin}\left(T_{0}^{i}\right) \wedge$ $\bigwedge_{1 \leq j \leq k_{i}} \operatorname{Inf}\left(T_{j}^{i}\right)$ and such that all $k_{i} \geq 1$. 


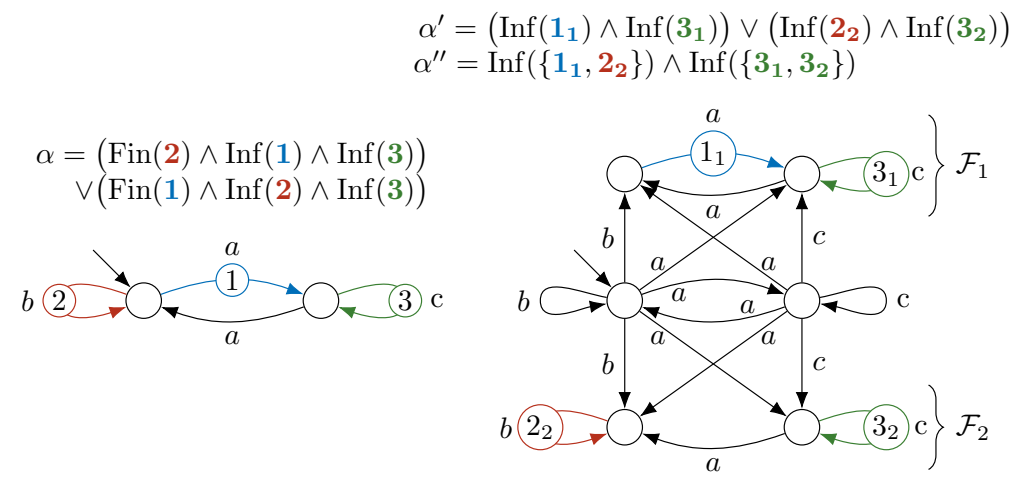

Fig. 1: Example of applying removeFin and removeFin ${ }_{\mathrm{GBA}}$ (Def. 3.4 to the automaton on the left. The result is the automaton on the right with acceptance $\alpha^{\prime}$ (removeFin), respectively $\alpha^{\prime \prime}$ (removeFin (rBA $)$.

The reason that a single Fin atom in each disjunct is enough is that $\operatorname{Fin}\left(T_{1}\right) \wedge$ $\operatorname{Fin}\left(T_{2}\right) \equiv_{\delta} \operatorname{Fin}\left(T_{1} \cup T_{2}\right)$ for all $T_{1}, T_{2}, \delta$. Taking $k_{i} \geq 1$ is also no restriction, as we can always add $\wedge \operatorname{Inf}(\delta)$ to any disjunct. Using standard Boolean operations one can transform a TELA with acceptance $\beta$ into DNF by just translating the acceptance formula into a formula $\alpha$ of the above form, with $|\alpha| \leq 2^{|\beta|}$.

Fin-Less Acceptance. To transform a TELA in DNF (see Def. 3.3) into an equivalent one without Fin-atoms we use the idea of 9,14 : a main copy of $\mathcal{A}$ is connected to one additional copy for each disjunct $\alpha_{i}$ of the acceptance condition, in which transitions from $T_{0}^{i}$ are removed. The acceptance condition ensures that every accepting run leaves the main copy eventually. Figure 1 shows an example.

Definition 3.4. Let $\mathcal{F}_{i}=\left(Q_{i}, \Sigma, \delta_{i}, I_{i}, \phi_{i}\right)$, where $Q_{i}=\left\{q^{(i)} \mid q \in Q\right\}, \delta_{i}=$ $\left\{\left(q^{(i)}, a, q^{(i)}\right) \mid\left(q, a, q^{\prime}\right) \in \delta \backslash T_{0}^{i}\right\}$ and $\phi_{i}=\bigwedge_{1 \leq j \leq k_{i}} \operatorname{Inf}\left(U_{j}^{i}\right)$, where $U_{j}^{i}=$ $\left\{\left(q^{(i)}, a, q^{\prime(i)}\right) \mid\left(q, a, q^{\prime}\right) \in T_{j}^{i} \backslash T_{0}^{i}\right\}$. Let $\operatorname{removeFin}(\mathcal{A})=\left(Q^{\prime}, \Sigma, \delta^{\prime}, I, \alpha^{\prime}\right)$ and $\operatorname{removeFin}_{\mathrm{GBA}}(\mathcal{A})=\left(Q^{\prime}, \Sigma, \delta^{\prime}, I, \alpha^{\prime \prime}\right)$, where $Q^{\prime}=Q \cup \bigcup_{1 \leq i \leq m} Q_{i}$ and:

- $\delta^{\prime}=\delta \cup \bigcup_{1 \leq i \leq m}\left(\delta_{i} \cup\left\{\left(q, a, q^{(i)}\right) \mid\left(q, a, q^{\prime}\right) \in \delta\right\}\right)$

- $\alpha^{\prime}=\bigvee_{1<i<m} \phi_{i}$

- $\alpha^{\prime \prime}=\bigwedge_{1 \leq j \leq k} \operatorname{Inf}\left(U_{j}^{1} \cup \ldots \cup U_{j}^{m}\right)$, with $k=\max _{i} k_{i}$ and $U_{j}^{i}=\delta_{i}$ if $k_{i}<j \leq k$.

Lemma 3.5. It holds that $\mathcal{L}(\mathcal{A})=\mathcal{L}(\operatorname{removeFin}(\mathcal{A}))=\mathcal{L}\left(\operatorname{removeFin}_{\mathrm{GBA}}(\mathcal{A})\right)$.

While removeFin $(\mathcal{A})$ is from [9, 14], removeFin $\operatorname{GBA}_{\mathrm{GB}}(\mathcal{A})$ is a variant that differs only in the acceptance and always produces GBA. Both consist of $m+1$ copies of $\mathcal{A}$ (with Fin-transitions removed). 


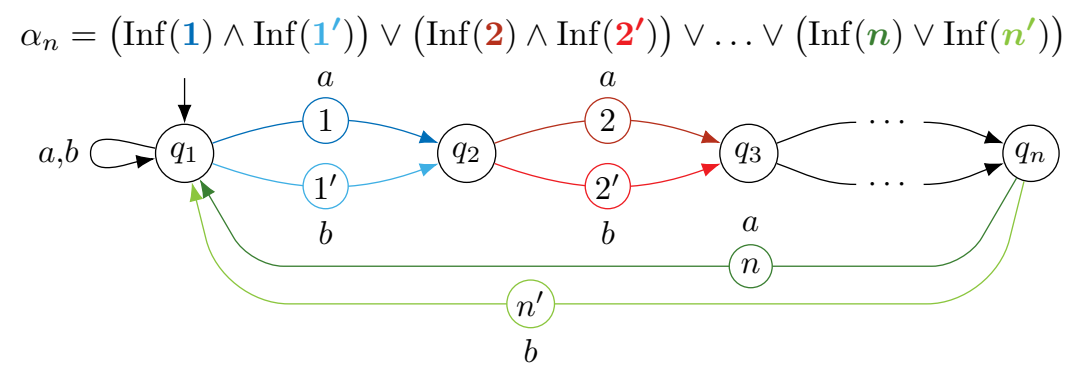

Fig. 2: A class of TELA where generating the CNF leads to $2^{n}$ many conjuncts.

\subsection{Construction of Generalized Büchi Automata}

Construction of Spot. The transformation from TELA to GBA from 14 is implemented in Sрот 15]. It transforms the automaton into DNF and then applies (an optimized version of) removeFin. The resulting fin-less acceptance condition is translated into conjunctive normal form $(\mathrm{CNF}) . \operatorname{As} \operatorname{Inf}\left(T_{1}\right) \vee \operatorname{Inf}\left(T_{2}\right) \equiv_{\delta}$ $\operatorname{Inf}\left(T_{1} \cup T_{2}\right)$ holds for all $\delta$, one can rewrite any fin-less condition in CNF into a conjunction of Inf-atoms, which is a generalized Büchi condition. When starting with a TELA $\mathcal{B}$ with acceptance $\beta$ and $N$ states, one gets a GBA with $N 2^{O(|\beta|)}$ states and $2^{O(|\beta|)}$ acceptance sets, as the fin-removal may introduce exponentially (in $|\beta|$ ) many copies, and the CNF may also be exponential in $|\beta|$.

Transforming a fin-less automaton into a GBA by computing the CNF has the advantage of only changing the acceptance condition, and in some cases it produces simple conditions directly. For example, Spot's TELA to GBA construction transforms a Rabin into a Büchi automaton, and a Streett automaton with $m$ acceptance pairs into a GBA with $m$ accepting sets. However, computing the CNF may also incur an exponential blow-up (Fig. 2 shows such an example).

Copy-based approaches. We now describe three approaches (remFin $\rightarrow \operatorname{split} \alpha$, split $\alpha \rightarrow$ remFin and remFin $\rightarrow$ rewrite $\alpha$ ), which construct GBA with at most $|\beta|$ acceptance sets. On the other hand, they generally produce automata with more states. They are based on 34 which first translates copies of $\mathcal{A}$ (corresponding to the disjuncts of the acceptance condition) to GBA, and then takes their sum. However, it is not specified in [34] how exactly Fin-atoms should be removed (they were concerned only with the theoretical complexity). We define:

$$
\begin{aligned}
\operatorname{remFin} \rightarrow \operatorname{split} \alpha(\mathcal{A}) & :=\bigoplus_{1 \leq i \leq m} \operatorname{GBA} \operatorname{split}(\operatorname{removeFin}(\mathcal{A}))[i] \\
\operatorname{split} \alpha \rightarrow \operatorname{remFin}(\mathcal{A}) & :=\bigoplus_{1 \leq i \leq m} \operatorname{GBA}_{\operatorname{lemoveFin}(\operatorname{split}(\mathcal{A})[i])} \operatorname{rem} \operatorname{rin}_{\mathrm{GBA}}(\mathcal{A})
\end{aligned}
$$


With removeFin as defined in Def. 3.4, the approaches remFin $\rightarrow$ split $\alpha$ and split $\alpha \rightarrow$ remFin produce the same automata (after removing non-accepting SCC's in remFin $\rightarrow$ split $\alpha$ ), and all three approaches create $O(m)$ copies of $\mathcal{A}$. Our implementation uses an optimized variant of removeFin, as provided by SPOT, which leads to different results for all three approaches.

\section{Determinization}

Determinization via single GBA. The standard way of determinizing TELA is to first construct a GBA, which is then determinized. Dedicated determinization procedures for GBA with $N$ states and $K$ acceptance sets produce deterministic Rabin automata with $2^{O(N(\log N+\log K))}$ states 36 . For a TELA $\mathcal{B}$ with $n$ states and acceptance $\beta$, the above translations yield GBA with $N=n 2^{O(|\beta|)}$ and $K=2^{O(|\beta|)}$ (SPOT's construction) or $N=n 2^{O(|\beta|)}$ and $K=O(|\beta|)$ (copy-based approaches). We evaluate the effect of the translations to GBA introduced in the previous chapter in the context of determinization in Sec. 6.

Determinization via a product construction. Another way to determinize a TELA $\mathcal{A}$ in DNF is to determinize the automata $\operatorname{split}(\mathcal{A})[i]$ one by one and then combining them with the disjunctive product construction of Def. 3.2

$$
\bigotimes_{1 \leq i \leq m} \operatorname{det}(\operatorname{removeFin}(\operatorname{split}(\mathcal{A})[i]))
$$

where "det" is any GBA-determinization procedure. Let $\mathcal{B}$ be a TELA with acceptance $\beta$ and $n$ states, and let $\alpha$ be an equivalent condition in DNF with $m$ disjuncts. Assuming an optimal GBA-determinization procedure, the product combines $m$ automata with $2^{O(n(\log n+\log |\beta|))}$ states and hence has $\left(2^{O(n(\log n+\log |\beta|))}\right)^{m}=2^{O\left(2^{|\beta|} \cdot n(\log n+\log |\beta|)\right)}$ states.

\section{Limit-deterministic TELA}

Limit-determinism has been studied mainly in the context of Büchi automata 12 . 37, 38, and we define it here for general TELA.

Definition 5.1. A TELA $\mathcal{A}=(Q, \Sigma, \delta, I, \alpha)$ is called limit-deterministic if there exists a partition $Q_{N}, Q_{D}$ of $Q$ such that

1. $\delta \cap\left(Q_{D} \times \Sigma \times Q_{N}\right)=\varnothing$,

2. for all $(q, a) \in Q_{D} \times \Sigma$ there exists at most one $q^{\prime}$ such that $\left(q, a, q^{\prime}\right) \in \delta$,

3. every accepting run $\rho$ of $\mathcal{A}$ satisfies $\inf (\rho) \cap\left(Q_{N} \times \Sigma \times Q_{N}\right)=\varnothing$.

This is a semantic definition and as checking emptiness of deterministic TELA is already coNP-hard, checking whether a TELA is limit-determinstic is also.

Proposition 5.2. Checking limit-determinism for TELA is coNP-complete.

An alternative syntactic definition for TELA in DNF, which implies limitdeterminism, is provided in Def. 5.3 . 
Definition 5.3. A TELA $\mathcal{A}=\left(Q, \Sigma, \delta,\left\{q_{0}\right\}, \alpha\right)$ in DNF, with $\alpha=\bigvee_{1<i<m} \alpha_{i}$, $\alpha_{i}=\operatorname{Fin}\left(T_{0}^{i}\right) \wedge \bigwedge_{1 \leq j \leq k_{i}} \operatorname{Inf}\left(T_{j}^{i}\right)$ and $k_{i} \geq 1$ for all $i$, is syntactically limitdeterministic if there exists a partition $Q_{N}, Q_{D}$ of $Q$ satisfying 1. and 2. of Def.5.1 and additionally $T_{j}^{i} \subseteq Q_{D} \times \Sigma \times Q_{D}$ for all $i \leq m$ and $1 \leq j \leq k_{i}$.

\section{$5.1 \quad$ Limit-determinization}

We first observe that replacing the product by a sum in the product-based determinization above yields limit-deterministic automata of single-exponential size (in contrast to the double-exponential lower-bound for determinization). Let $\mathcal{A}$ be a TELA in DNF with $n$ states and acceptance $\alpha=\bigvee_{1 \leq i \leq m} \alpha_{i}$, where $\alpha_{i}=\operatorname{Fin}\left(T_{0}^{i}\right) \wedge \bigwedge_{1 \leq j \leq k_{i}} \operatorname{Inf}\left(T_{j}^{i}\right)$ (see Def. 3.3), and let $\mathcal{A}_{i}=\operatorname{split}(\overline{\mathcal{A}})[i]$.

Proposition 5.4. $\bigoplus_{1 \leq i \leq m} \operatorname{det}\left(\operatorname{removeFin}\left(\mathcal{A}_{i}\right)\right)$ is limit-deterministic and of size $\sum_{1 \leq i \leq m}\left|\operatorname{det}\left(\mathcal{A}_{i}\right)\right|=m \cdot 2^{O(n(\log n+\log k))}$, where $k=\max \left\{k_{i} \mid 1 \leq i \leq m\right\}$.

If "det" is instantiated by a GBA-determinization that produces Rabin automata, then the result is in DNF and syntactically limit-determinstic. Indeed, in this case the only nondeterminism is the choice of the initial state. But "det" can, in principle, also be replaced by any limit-determinization procedure for GBA.

We now extend the limit-determinization constructions of 12 (for Büchi automata) and [6, 7, 19] (for GBA) to Emerson-Lei conditions in DNF. These constructions use an initial component and an accepting breakpoint component 27 for $\mathcal{A}$, which is deterministic. The following construction differs in two ways: there is one accepting component per disjunct of the acceptance condition, and the accepting components are constructed from $\mathcal{A}$ without considering the Fintransitions of that disjunct. To define the accepting components we use the subset transition function $\theta$ associated with $\delta: \theta(P, a)=\bigcup_{q \in P}\left\{q^{\prime} \mid\left(q, a, q^{\prime}\right) \in \delta\right\}$ for $(P, a) \in 2^{Q} \times \Sigma$, and additionally we define $\left.\theta\right|_{T}(P, a)=\bigcup_{q \in P}\left\{q^{\prime} \mid\left(q, a, q^{\prime}\right) \in\right.$ $\delta \cap T\}$. These functions are extended to finite words in the standard way.

Definition 5.5. Let $\theta_{i}=\left.\theta\right|_{\delta \backslash T_{0}^{i}}$ and define $\mathcal{B} \mathcal{P}_{i}=\left(Q_{i}, \Sigma, \delta_{i},\left\{p_{0}\right\}, \operatorname{Inf}\left(\delta_{i}^{\text {break }}\right)\right)$ with: $Q_{i}=\left\{(R, B, l) \in 2^{Q} \times 2^{Q} \times\left\{0, \ldots, k_{i}\right\} \mid B \subseteq R\right\}, p_{0}=(I, \varnothing, 0)$ and

$$
\begin{aligned}
& \delta_{i}^{\text {main }}=\left\{\left(\left(R_{1}, B_{1}, l\right), a,\left(R_{2}, B_{2}, l\right)\right) \mid \begin{array}{l}
R_{2}=\theta_{i}\left(R_{1}, a\right), \\
B_{2}=\left.\theta_{i}\left(B_{1}, a\right) \cup \theta_{i}\right|_{T_{l}^{i}}\left(R_{1}, a\right)
\end{array}\right\} \\
& \delta_{i}^{\text {break }}=\left\{\begin{array}{ll}
\left(\left(R_{1}, B_{1}, l\right), a,\left(R_{2}, \varnothing, l^{\prime}\right) \mid\right. & \left(\left(R_{1}, B_{1}, l\right), a,\left(R_{2}, B_{2}, l\right)\right) \in \delta_{i}^{\text {main }}, \\
l_{2}=(l+1) \bmod \left(k_{i}+1\right)
\end{array}\right\} \\
& \delta_{i}=\left\{\left(\left(R_{1}, B_{1}, l\right), a,\left(R_{2}, B_{2}, l\right)\right) \in \delta_{i}^{\text {main }} \mid R_{2} \neq B_{2}\right\} \cup \delta_{i}^{\text {break }}
\end{aligned}
$$

In state $(R, B, l)$, intuitively $R$ is the set of states reachable for the prefix word in $\mathcal{A}$ without using transitions from $T_{0}^{i}$, while $B$ are the states in $R$ which have seen a transition in $T_{l}^{i}$ since the last "breakpoint". The breakpoint-transitions are $\delta_{i}^{\text {break }}$, which occur when all states in $R$ have seen an accepting transition 
since the last breakpoint (namely if $R=B$ ). The breakpoint construction underapproximates the language of a given GBA, in general.

We define two limit-deterministic Büchi automata (LDBA) $\mathcal{G}_{\mathcal{A}}^{\mathrm{LD}}$ and $\mathcal{G}_{\mathcal{A}}^{\mathrm{GFM}}$ where $\mathcal{G}_{\mathcal{A}}^{\mathrm{GFM}}$ is additonally good-for-MDP $(\mathrm{GFM})\left[20\right.$. This means that $\mathcal{G}_{\mathcal{A}}^{\mathrm{G} F M}$ can be used to solve certain quantitative probabilistic model checking problems (see Sec. 5.2). Both use the above breakpoint automata as accepting components. While $\mathcal{G}_{\mathcal{A}}^{\mathrm{LD}}$ simply uses a copy of $\mathcal{A}$ as initial component, $\mathcal{G}_{\mathcal{A}}^{\mathrm{GFM}}$ uses the deterministic subset-automaton of $\mathcal{A}$ (it resembles the cut-deterministic automata of [6]). Furthermore, to ensure the GFM property, there are more transitions between initial and accepting copies in $\mathcal{G}_{\mathcal{A}}^{\mathrm{GFM}}$. The construction of $\mathcal{G}_{\mathcal{A}}^{\mathrm{GFM}}$ extends the approach for GBA in [19] (also used for probabilistic model checking) to TELA. We will distinguish elements from sets $Q_{i}$ for different $i$ from Def. 5.5 by using subscripts (e.g. $\left.(R, P, l)_{i}\right)$ and assume that these sets are pairwise disjoint.

Definition $5.6\left(\mathcal{G}_{\mathcal{A}}^{\mathrm{LD}}\right.$ and $\left.\mathcal{G}_{\mathcal{A}}^{\mathrm{GFM}}\right)$. Let $Q_{\mathrm{acc}}=\bigcup_{1 \leq i \leq m} Q_{i}, \delta_{\mathrm{acc}}=\bigcup_{1 \leq i \leq m} \delta_{i}$ and $\alpha_{\text {acc }}=\operatorname{Inf}\left(\bigcup_{1 \leq i \leq m} \delta_{i}^{\text {break }}\right)$. Define

$$
\mathcal{G}_{\mathcal{A}}^{\mathrm{LD}}=\left(Q \cup Q_{\mathrm{acc}}, \Sigma, \delta^{\mathrm{LD}}, I, \alpha^{\prime}\right) \text { and } \mathcal{G}_{\mathcal{A}}^{\mathrm{GFM}}=\left(2^{Q} \cup Q_{\mathrm{acc}}, \Sigma, \delta^{\mathrm{GFM}},\{I\}, \alpha^{\prime}\right)
$$

with

$$
\begin{aligned}
\delta^{\mathrm{LD}} & =\delta \cup \delta_{\text {bridge }}^{\mathrm{LD}} \cup \delta_{\mathrm{acc}} \quad \text { and } \quad \delta^{\mathrm{GFM}}=\theta \cup \delta_{\text {bridge }}^{\mathrm{GFM}} \cup \delta_{\text {acc }} \\
\delta_{\text {bridge }}^{\mathrm{LD}} & =\left\{\left(q, a,\left(\left\{q^{\prime}\right\}, \varnothing, 0\right)_{i}\right) \mid\left(q, a, q^{\prime}\right) \in \delta \text { and } 1 \leq i \leq m\right\} \\
\delta_{\text {bridge }}^{\mathrm{GFM}} & =\left\{\left(P, a,\left(P^{\prime}, \varnothing, 0\right)_{i}\right) \mid P^{\prime} \subseteq \theta(P, a) \text { and } 1 \leq i \leq m\right\}
\end{aligned}
$$

As $\delta_{i}^{\text {break }} \subseteq \delta_{\text {acc }}$ for all $i$, both $\mathcal{G}_{\mathcal{A}}^{\mathrm{LD}}$ and $\mathcal{G}_{\mathcal{A}}^{\mathrm{GFM}}$ are syntactically limit-deterministic. The proofs of correctness are similar to ones of the corresponding constructions for GBA 6, Thm. 7.6]. We show later in Prop. 5.14 that $\mathcal{G}_{\mathcal{A}}^{\mathrm{GFM}}$ is GFM.

Theorem 5.7. $\mathcal{G}_{\mathcal{A}}^{\mathrm{LD}}$ and $\mathcal{G}_{\mathcal{A}}^{\mathrm{GFM}}$ are syntactically limit-deterministic and satisfy $\mathcal{L}\left(\mathcal{G}_{\mathcal{A}}^{\mathrm{LD}}\right)=\mathcal{L}\left(\mathcal{G}_{\mathcal{A}}^{\mathrm{GFM}}\right)=\mathcal{L}(\mathcal{A})$. Their number of states is in $O\left(n+3^{n} m k\right)$ for $\mathcal{G}_{\mathcal{A}}^{\mathrm{LD}}$ and $O\left(2^{n}+3^{n} m k\right)=O\left(|\alpha|^{2} \cdot 3^{n}\right)$ for $\mathcal{G}_{\mathcal{A}}^{\mathrm{GFM}}$, where $k=\max \left\{k_{i} \mid 1 \leq i \leq m\right\}$.

Corollary 5.8. Given TELA $\mathcal{B}$ (not necessarily in DNF) with acceptance condition $\beta$ and $N$ states, there exists an equivalent LDBA with $2^{O(|\beta|+N)}$ states.

\subsection{Probabilistic model checking}

We now discuss how these constructions can be used for probabilistic model checking. First, consider the qualitative model checking problem to decide $\operatorname{Pr}_{\mathcal{M}}^{\max }(\mathcal{L}(\mathcal{A}))>0$, under the assumption that $\mathcal{A}$ is a limit-deterministic TELA. While NP-hardness follows from the fact that the problem is already hard for deterministic TELA [28, Thm. 5.13], we now show that it is also in NP. Furthermore, it is in $\mathrm{P}$ for automata with a fin-less acceptance condition. This was already known for LDBA [12, and our proof uses similar arguments.

Proposition 5.9. Deciding $\operatorname{Pr}_{\mathcal{M}}^{\max }(\mathcal{L}(\mathcal{A}))>0$, given an $M D P \mathcal{M}$ and a limitdeterministic TELA $\mathcal{A}$, is NP-complete. If $\mathcal{A}$ has a fin-less acceptance condition, then the problem is in $P$. 
Now we show that $\mathcal{G}_{\mathcal{A}}^{\mathrm{GFM}}$ is good-for-MDP 20]. In order to define this property, we introduce the product of an MDP with a nondeterministic automaton in which, intuitively, the scheduler is forced to resolve the nondeterminism by choosing the next state of the automaton (see $[20,22]$ ). We assume that the automaton used to build the product has a single initial state, which holds for $\mathcal{G}_{\mathcal{A}}^{\mathrm{GFM}}$.

Definition 5.10. Given an $M D P \mathcal{M}=\left(S, s_{0}\right.$, Act, $\left.P, \Sigma, L\right)$ and TELA $\mathcal{G}=$ $\left(Q, \Sigma, \delta,\left\{q_{0}\right\}, \alpha\right)$ we define the $M D P \mathcal{M} \times \mathcal{G}=\left(S \times Q,\left(s_{0}, q_{0}\right)\right.$, Act $\left.\times Q, P^{\times}, \Sigma, L^{\times}\right)$ with $L^{\times}((s, q))=L(s)$ and

$$
P^{\times}\left((s, q),(\alpha, p),\left(s^{\prime}, q^{\prime}\right)\right)= \begin{cases}P\left(s, \alpha, s^{\prime}\right) & \text { if } p=q^{\prime} \text { and }\left(q, L(s), q^{\prime}\right) \in \delta \\ 0 & \text { otherwise }\end{cases}
$$

We define the accepting paths $\Pi_{a c c}$ of $\mathcal{M} \times \mathcal{G}$ to be:

$$
\Pi_{a c c}=\left\{\left(s_{0}, q_{0}\right) \alpha_{0}\left(s_{1}, q_{1}\right) \alpha_{1} \ldots \in \operatorname{Paths}(\mathcal{M} \times \mathcal{G}) \mid q_{0}, L\left(s_{0}\right), q_{1}, L\left(s_{1}\right) \ldots \models \alpha\right\}
$$

A Büchi automaton $\mathcal{G}$ is good-for-MDP $(\mathrm{GFM})$ if $\operatorname{Pr}_{\mathcal{M}}^{\max }(\mathcal{L}(\mathcal{G}))=\operatorname{Pr}_{\mathcal{M} \times \mathcal{G}}^{\max }\left(\Pi_{a c c}\right)$ holds for all MDP $\mathcal{M}[20$. The inequality " $\geq$ " holds for all automata [22, Thm. 1], but the other direction requires, intuitively, that a scheduler on $\mathcal{M} \times \mathcal{G}$ is able to safely resolve the nondeterminism of the automaton based on the prefix of the run. This is trivially satisfied by deterministic automata, but good-for-games automata also have this property 22$]$. Limit-deterministic Büchi automata are not GFM in general, for example, $\mathcal{G}_{\mathcal{A}}^{\mathrm{LD}}$ may not be (see Ex. 5.12).

We fix an arbitrary MDP $\mathcal{M}$ and show that $\operatorname{Pr}_{\mathcal{M}}^{\max }(\mathcal{L}(\mathcal{A})) \leq \mathbf{P r}_{\mathcal{M} \times \mathcal{G}_{\mathcal{A}}^{\mathrm{GFM}}}^{\max }\left(\Pi_{a c c}\right)$. To this end we show that for any finite-memory scheduler $\mathfrak{S}$ on $\mathcal{M}^{\mathcal{A}}$ we find a scheduler $\mathfrak{S}^{\prime}$ on $\mathcal{M} \times \mathcal{G}_{\mathcal{A}}^{\mathrm{GFM}}$ such that $\operatorname{Pr}_{\mathcal{M}}^{\mathfrak{S}}(\mathcal{L}(\mathcal{A})) \leq \operatorname{Pr}_{\mathcal{M} \times \mathcal{G}_{\mathcal{A}}^{\mathrm{GFM}}}^{\mathfrak{S}^{\prime}}\left(\Pi_{a c c}\right)$. The restriction to finite-memory schedulers is allowed because the maximal probability to satisfy an $\omega$-regular property is always attained by such a scheduler 4, Secs. 10.6.3 and 10.6.4]. Let $\mathcal{M}_{\mathfrak{S}} \times \mathcal{D}$ be the product of the induced finite Markov chain $M_{\mathfrak{S}}$ with $\mathcal{D}=\bigotimes_{1<i<m} \mathcal{D}_{i}$, where $\mathcal{D}_{i}=\operatorname{det}(\operatorname{removeFin}(\operatorname{split}(\mathcal{A})[i]))$ and "det" is the GBA-determinization procedure from 36, which makes $\mathcal{D}$ a deterministic Rabin automaton. The scheduler $\mathfrak{S}^{\prime}$ is constructed as follows. It stays inside the initial component of $\mathcal{M} \times \mathcal{G}_{\mathcal{A}}^{\mathrm{GFM}}$ and mimics the action chosen by $\mathfrak{S}$ until the corresponding path in $\mathcal{M}_{\mathfrak{S}} \times \mathcal{D}$ reaches an accepting bottom strongly connected component (BSCC) $B$. This means that the transitions of $\mathcal{D}$ induced by $B$ satisfy one of the Rabin pairs. The following lemma shows that in this case there exists a state in one of the breakpoint components to which $\mathfrak{S}^{\prime}$ can safely move.

Lemma 5.11. Let $\mathfrak{s}$ be a state in an accepting $B S C C B$ of $\mathcal{M}_{\mathfrak{S}} \times \mathcal{D}$ and $\pi_{1}$ be a finite path that reaches $\mathfrak{s}$ from the initial state of $\mathcal{M}_{\mathfrak{S}} \times \mathcal{D}$. Then, there exists $1 \leq i \leq m$ and $Q^{\prime} \subseteq \theta\left(I, L\left(\pi_{1}\right)\right)$ such that:

$$
\operatorname{Pr}_{\mathfrak{s}}\left(\left\{\pi \mid L(\pi) \text { is accepted from }\left(Q^{\prime}, \varnothing, 0\right) \text { in } \mathcal{B} \mathcal{P}_{i}\right\}\right)=1
$$

The lemma does not hold if we restrict ourselves to singleton $\{q\} \subseteq \theta\left(I, L\left(\pi_{1}\right)\right)$ (see Ex. 5.12). Hence, restricting $\delta_{\text {bridge }}^{\text {GFM }}$ to such transitions (as for $\delta_{\text {bridge }}^{\mathrm{LD}}$, see Def. 5.6 would not guarantee the GFM property. 


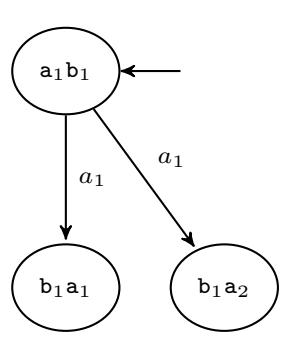

(a)

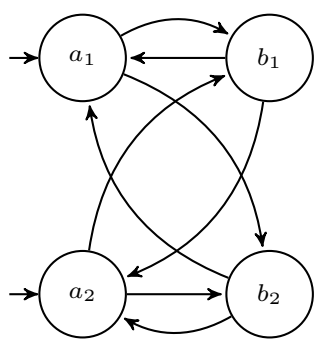

(b)

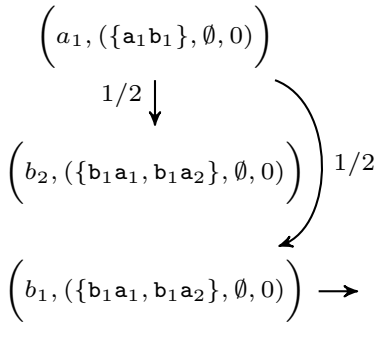

(c)

Fig. 3: Restricting $\delta_{\text {bridge }}^{\mathrm{GFM}}$ to transitions with endpoints of the form $(s,(\{q\}, \varnothing, 0))$ (similar to $\delta_{\text {bridge }}^{\mathrm{LD}}$ ) would not guarantee the GFM property (see Ex. 5.12p.

Example 5.12. Consider the automaton $\mathcal{A}$ with states $\left\{\mathrm{a}_{i} \mathrm{~b}_{j} \mid i, j \in\{1,2\}\right\} \cup$ $\left\{\mathrm{b}_{i} \mathrm{a}_{j} \mid i, j \in\{1,2\}\right\}$, where $\mathrm{a}_{i} \mathrm{~b}_{j}$ has transitions labeled by $a_{i}$ to $\mathrm{b}_{j} \mathrm{a}_{1}$ and $\mathrm{b}_{j} \mathrm{a}_{2}$. Transitions of states $\mathrm{b}_{i} \mathrm{a}_{j}$ are defined analogously, and all states in $\left\{\mathrm{a}_{i} \mathrm{~b}_{j} \mid i, j \in\right.$ $\{1,2\}\}$ are initial (Fig. 3 a shows the transitions of $a_{1} b_{1}$ ). All transitions are accepting for a single Büchi condition, and hence $\mathcal{L}(\mathcal{A})=\left(\left\{a_{i} b_{j} \mid i, j \in\{1,2\}\right\}\right)^{\omega}$.

Consider the Markov chain $\mathcal{M}$ in Fig. $3 \mathrm{~b}$ (transition probabilities are all $1 / 2$ and ommitted in the figure). Clearly, $\operatorname{Pr}_{\mathcal{M}}(\mathcal{L}(\mathcal{A}))=1$. Fig. 3c shows a part of the product of $\mathcal{M}$ with the breakpoint automaton $\mathcal{B P}$ for $\mathcal{A}$ (Def. 5.5) starting from $\left(a_{1},\left(\left\{\mathrm{a}_{1} \mathrm{~b}_{1}\right\}, \varnothing, 0\right)\right)$. The state $\left(b_{2},\left(\left\{\mathrm{~b}_{1} \mathrm{a}_{1}, \mathrm{~b}_{1} \mathrm{a}_{2}\right\}, \varnothing, 0\right)\right)$ is a trap state as $\mathrm{b}_{1} \mathrm{a}_{1}$ and $\mathrm{b}_{1} \mathrm{a}_{2}$ have no $b_{2}$-transition. Hence, $\left(a_{1},\left(\left\{\mathrm{a}_{1} \mathrm{~b}_{1}\right\}, \varnothing, 0\right)\right)$ generates an accepting path with probability at most $1 / 2$. This is true for all states $\left(s,\left(P^{\prime}, \varnothing, 0\right)\right)$ of $\mathcal{M} \times \mathcal{B P}$ where $P^{\prime}$ is a singleton. But using $\delta_{\text {bridge }}^{\mathrm{LD}}$ to connect initial and accepting components implies that any accepting path sees such a state. Hence, using $\delta_{\text {bridge }}^{\mathrm{LD}}$ to define $\mathcal{G}_{\mathcal{A}}^{\mathrm{GFM}}$ would not guarantee the GFM property.

Using Lem. 5.11 we can define $\mathfrak{S}^{\prime}$ such that the probability accepting paths under $\mathfrak{S}^{\prime}$ in $\mathcal{M} \times \mathcal{G}_{\mathcal{A}}^{\mathrm{GFM}}$ is at least as high as that of paths with label in $\mathcal{L}(\mathcal{A})$ in $\mathcal{M}_{\mathfrak{S}}$. This is the non-trivial direction of the GFM property.

Lemma 5.13. For every finite-memory scheduler $\mathfrak{S}$ on $\mathcal{M}$, there exists a scheduler $\mathfrak{S}^{\prime}$ on $\mathcal{M} \times \mathcal{G}_{\mathcal{A}}^{\mathrm{GFM}}$ such that:

$$
\operatorname{Pr}_{\mathcal{M} \times \mathcal{G}_{\mathcal{A}}^{\mathrm{GFM}}}^{\mathfrak{S}^{\prime}}\left(\Pi_{a c c}\right) \geq \operatorname{Pr}_{\mathcal{M}}^{\mathfrak{S}}(\mathcal{L}(\mathcal{A}))
$$

Proposition 5.14. The automaton $\mathcal{G}_{\mathcal{A}}^{\mathrm{GFM}}$ is good-for-MDP.

To compute $\operatorname{Pr}_{\mathcal{M}}^{\max }(\mathcal{L}(\mathcal{B}))$ one can translate $\mathcal{B}$ into an equivalent TELA $\mathcal{A}$ in DNF, then construct $\mathcal{G}_{\mathcal{A}}^{\mathrm{GFM}}$ and finally compute $\operatorname{Pr}_{\mathcal{M} \times \mathcal{G}^{\mathrm{GFM}}}^{\max }\left(\Pi_{\text {acc }}\right)$. The automaton $\mathcal{G}_{\mathcal{A}}^{\mathrm{GFM}}$ is single-exponential in the size of $\mathcal{B}$ by Thm. 5.7 and $\operatorname{Pr}_{\mathcal{M} \times \mathcal{G}_{\mathcal{A}}^{\mathrm{GFM}}}^{\max }\left(\Pi_{\mathrm{acc}}\right)$ can be computed in polynomial time in the size of $\mathcal{M} \times \mathcal{G}_{\mathcal{A}}^{\mathrm{GFM}}$ [4. Thm. 10.127].

Theorem 5.15. Given a TELA $\mathcal{B}$ (not necessarily in DNF) and an $M D P \mathcal{M}$, the value $\operatorname{Pr}_{\mathcal{M}}^{\max }(\mathcal{L}(\mathcal{B}))$ can be computed in single-exponential time. 


\section{Experimental evaluation}

The product approach combines a sequence of deterministic automata using the disjunctive product. We introduce the langcover heuristic: the automata are "added" to the product one by one, but only if their language is not already subsumed by the automaton constructed so far. This leads to substantially smaller automata in many cases, but is only efficient if checking inclusion for the considered automata types is efficent. In our case this holds (the automata are deterministic with a disjunction of parity conditions as acceptance), but it is not the case for arbitrary deterministic TELA, or nondeterministic automata.

Implementation. We compare the following implementations of the constructions discussed above ${ }^{1}$ SPot uses the TELA to GBA translator of SpOT, simplifies (using SPOT's postprocessor with preference Small) and degeneralizes the result and then determinizes using a version of Safra's algorithm [15, 32. The removeFin function that is used is an optimized version of Def. 3.4. In remFin $\rightarrow$ split $\alpha$, split $\alpha \rightarrow$ remFin and remFin $\rightarrow$ rewrite $\alpha$, the first step is replaced by the corresonding TELA to GBA construction (using SPOT's removeFin). The product approach (also implemented using the SPOT-library) is called product and product (no langcover) (without the langcover heuristic). The intermediate GBA are also simplified. The construction $\mathcal{G}_{\mathcal{A}}^{\mathrm{LD}}$ is implemented in limit-det., using the Spot-library and parts of SEmINaTOR. We compare it to limit-det. via GBA, which concatenates the TELA to GBA construction of SPоT with the limitdeterminization of SEMINATOR. Similarly, good-for-MDP and good-for-MDP via GBA are the construction $\mathcal{G}_{\mathcal{A}}^{\mathrm{GFM}}$ applied to $\mathcal{A}$ directly, or to the GBA as constructed by SpOT. Both constructions via GBA are in the worst case double-exponential. No post-processing is applied to any output automaton.

Experiments. Computations were performed on a computer with two Intel E5-2680 CPUs with 8 cores each at $2.70 \mathrm{GHz}$ running Linux. Each individual experiment was limited to a single core, 15 GB of memory and 1200 seconds. We use versions 2.9.4 of Spot (configured to allow 256 acceptance sets) and 2.0 of SEminator.

Our first benchmark set (called random) consists of 1000 TELA with 4 to 50 states and 8 sets of transitions $T_{1}, \ldots, T_{8}$ used to define the acceptance conditions. They are generated using SPоT's procedure random_graph() by specifying probabilities such that: a triple $\left(q, a, q^{\prime}\right) \in Q \times \Sigma \times Q$ is included in the transition relation $(3 /|Q|)$ and such that a transition $t$ is included in a set $T_{j}$ $(0.2)$. We use only transition systems that are nondeterministic. The acceptance condition is generated randomly using SPOT's procedure acc_code: :random(). We transform the acceptance condition to DNF and keep those acceptance conditions whose lengths range between 2 and 21 and consist of at least two disjuncts. To quantify the amount of nondeterminism, we divide the number of pairs of transitions of the form $\left(q, a, q_{1}\right),\left(q, a, q_{2}\right)$, with $q_{1} \neq q_{2}$, of the automaton by its number of states.

\footnotetext{
$\overline{1}$ The source code and data of all experiments are available at 21 .
} 
Table 1: Evaluation of benchmarks random and DNF. Columns "states", "time" and "acceptance" refer to the respective median values, where mem-/timeouts are counted as larger than the rest. Values in brackets refer to the subset of input automata for which at least one determinization needed more than 0.5 seconds (447 (182) automata for benchmark random (DNF)).

\begin{tabular}{|c|c|c|c|c|c|c|c|c|}
\hline & algorithm & timeouts & memouts & states & time & acceptance & $\begin{array}{l}\text { interm } \\
\text { states }\end{array}$ & $\begin{array}{l}\text { ediate GBA } \\
\text { acceptance }\end{array}$ \\
\hline \multirow{10}{*}{$\begin{array}{l}\text { हूँ } \\
\text { हूँ }\end{array}$} & SPOT & $0.5 \%$ & $9.9 \%$ & $3,414(59,525)$ & $<1(1.5)$ & $10(17)$ & 71 & 2 \\
\hline & remFin $\rightarrow$ split $\alpha$ & $0.5 \%$ & $15.2 \%$ & $8,639(291,263)$ & $<1(9.7)$ & $14(24)$ & 109 & 2 \\
\hline & split $\alpha \rightarrow$ remFin & $0.7 \%$ & $17.8 \%$ & $14,037(522,758)$ & $<1(21.0)$ & $14(24)$ & 119 & 2 \\
\hline & remFin $\rightarrow$ rewrite $\alpha$ & $1.6 \%$ & $18.7 \%$ & $15,859(1,024,258)$ & $<1(40.2)$ & $14(26)$ & 116 & 2 \\
\hline & product & $1.3 \%$ & $7.9 \%$ & $3,069(43,965)$ & $<1(1.2)$ & $18(29)$ & & \\
\hline & product (no langcover) & $0.7 \%$ & $9.0 \%$ & $3,857(109,908)$ & $<1(1.1)$ & $24(38)$ & & \\
\hline & limit-det. & $0.0 \%$ & $0.0 \%$ & $778(3,346)$ & $<1(<1)$ & $1(1)$ & & \\
\hline & limit-det. via GBA & $1.6 \%$ & $0.3 \%$ & $463(1,556)$ & $<1(1.6)$ & $1(1)$ & & \\
\hline & good-for-MDP & $9.3 \%$ & $13.4 \%$ & $5,069(192.558)$ & $2.0(139.6)$ & $1(1)$ & & \\
\hline & good-for-MDP via GBA & $5.5 \%$ & $44.0 \%$ & $71,200(-)$ & $836.9(-)$ & $1(-)$ & & \\
\hline \multirow{2}{*}{$\begin{array}{l} \\
\vdots \\
a\end{array}$} & SPOT & $0.4 \%$ & $6.2 \%$ & 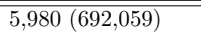 & < <1(18.3) & $111(25)$ & 30 & 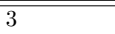 \\
\hline & product & $0.0 \%$ & $3.8 \%$ & $2,596(114,243)$ & $<1(4.6)$ & $13(24)$ & & \\
\hline
\end{tabular}

Tab. 1 shows that the product produces smallest deterministic automata overall. SPOT produces best results among the algorithms that go via a single GBA. One reason for this is that after GBA-simplifications of Spot, the number of acceptance marks of the intermediate GBA are comparable. Figure 4 (left) compares SPOT and product and partitions the input automata according to acceptance complexity (measured in the size of their DNF) and amount of nondeterminism. Each subset of input automata is of roughly the same size (159-180) (see Tab. 2 in the appendix). The graph depicts the median of the ratio (product / SPOT) for the measured values. For time- or memouts of SPOT (product) we define the ratio as $0(\infty)$. If both failed, the input is discarded. The number of time- and memouts grows with the amount of nondeterminism and reaches up to $42 \%$. The approach product performs better for automata with more nondeterminism and complex acceptance conditions as the results have fewer states and the computation times are smaller compared to Sрот.

The limit-deterministic automata are generally much smaller than the deterministic ones, and limit-det via GBA . performs best in this category. However, the construction $\mathcal{G}_{\mathcal{A}}^{\mathrm{LD}}$ (limit-det.) resulted in fewer time- and memouts.

For GFM automata we see that computing $\mathcal{G}_{\mathcal{A}}^{\mathrm{GFM}}$ directly, rather than first computing a GBA, yields much better results (good-for-MDP vs. good-for-MDP via GBA). However, the GFM automata suffer from significantly more time- and memouts than the other approaches. The automata sizes are comparable on average with SPOT's determinization (see Fig. 7 in the appendix). Given their similarity to the pure limit-determinization constructions, and the fact that their acceptance condition is much simpler than for the deterministic automata, we believe that future work on optimizing this construction could make it a competitive alternative for probabilistic model checking using TELA. 

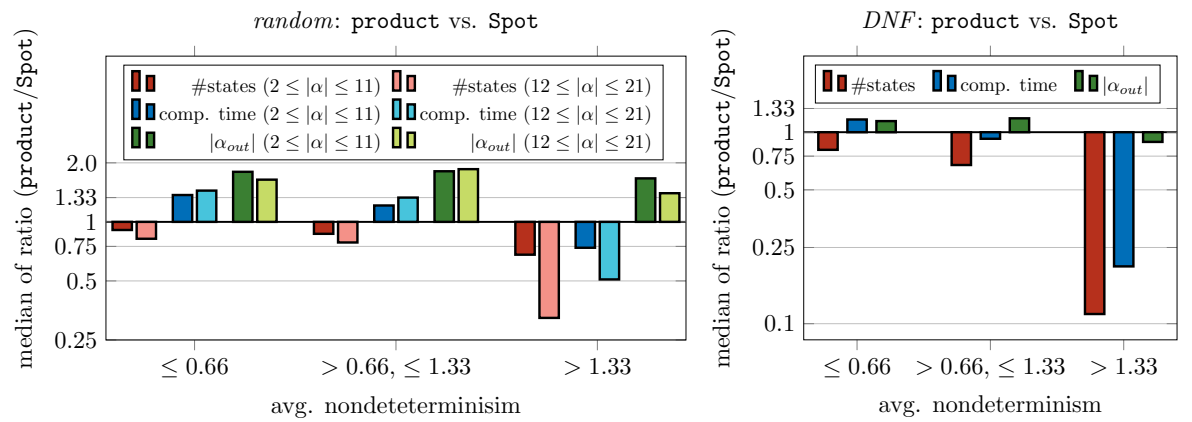

Fig. 4: Comparison of SPOT and product, with input automata grouped by the size of the DNF of their acceptance condition and the amount of nondeterminism.

The second benchmark (called DNF) consists of 500 TELA constructed randomly as above, apart from the acceptance conditions. They are in DNF with 2-3 disjuncts, with 2-3 Inf-atoms and 0-1 Fin-atoms each (all different). Such formulas tend to lead to larger CNF conditions, which benefits the new approaches. Fig. 4 (right) shows the median ratio of automata sizes, computation times and acceptance sizes, grouped by the amount of nondeterminism. We do not consider different lengths of acceptance conditions because the subsets of input automata are already relatively small (140-193). Again, product performs better for automata with more nondeterminism.

\section{Conclusion}

We have introduced several new approaches to determinize and limit-determinize automata under the Emerson-Lei acceptance condition. The experimental evaluation shows that in particular the product approach performs very well. Furthermore, we have shown that the complexity of limit-determinizing TELA is single-exponential (in contrast to the double-exponential blow-up for determinization). One of our constructions produces limit-deterministic good-for-MDP automata, which can be used for quantitative probabilistic verification.

This work leads to several interesting questions. The presented constructions would benefit from determinization procedures for GBA which trade a general acceptance condition (rather than Rabin or parity) for a more compact state-space of the output. Similarly, translations from LTL to compact, nondeterministic TELA would allow them to be embedded into (probabilistic) model-checking tools for LTL (a first step in this direction is made in [26]). It would be interesting to study, in general, what properties can be naturally encoded directly into nondeterministic TELA. Another open point is to evaluate the good-for-MDP automata in the context of probabilistic model checking in practice.

Acknowledgments. We thank David Müller for suggesting to us the problem of determinizing Emerson Lei automata and many discussions on the topic. 


\section{References}

1. Allen Emerson, E., Lei, C.L.: Modalities for model checking: Branching time logic strikes back. Science of Computer Programming 8(3), 275-306 (Jun 1987). https://doi.org/10.1016/0167-6423(87)90036-0

2. Babiak, T., Blahoudek, F., Duret-Lutz, A., Klein, J., Křetínský, J., Müller, D., Parker, D., Strejček, J.: The Hanoi Omega-Automata Format. In: Kroening, D., Păsăreanu, C.S. (eds.) Computer Aided Verification. pp. 479-486. Lecture Notes in Computer Science, Springer International Publishing, Cham (2015). https://doi.org/10.1007/978-3-319-21690-4_31

3. Baier, C., Blahoudek, F., Duret-Lutz, A., Klein, J., Müller, D., Strejček, J.: Generic Emptiness Check for Fun and Profit. In: Chen, Y.F., Cheng, C.H., Esparza, J. (eds.) Automated Technology for Verification and Analysis. pp. 445-461. Lecture Notes in Computer Science, Springer International Publishing, Cham (2019). https://doi.org/10.1007/978-3-030-31784-3_26

4. Baier, C., Katoen, J.P.: Principles of Model Checking (Representation and Mind Series). The MIT Press (2008)

5. Ben-Ari, M.: Principles of the Spin Model Checker. Springer-Verlag, London (2008). https://doi.org/10.1007/978-1-84628-770-1

6. Blahoudek, F.: Automata for Formal Methods: Little Steps Towards Perfection Ph.D. thesis, Masaryk University, Faculty of Informatics (2018), https://is.muni cz/th/gwriw/?lang=en

7. Blahoudek, F., Duret-Lutz, A., Klokočka, M., Křetínský, M., Strejček, J.: Seminator: A Tool for Semi-Determinization of Omega-Automata. In: EPiC Series in Computing. vol. 46, pp. 356-367. EasyChair (May 2017). https://doi.org/10.29007/k5nl

8. Blahoudek, F., Major, J., Strejček, J.: LTL to Smaller Self-Loop Alternating Automata and Back. In: Hierons, R.M., Mosbah, M. (eds.) Theoretical Aspects of Computing - ICTAC 2019. pp. 152-171. Lecture Notes in Computer Science, Springer International Publishing, Cham (2019). https://doi.org/10.1007/978-3030-32505-3_10

9. Bloemen, V., Duret-Lutz, A., van de Pol, J.: Model checking with generalized Rabin and Fin-less automata. International Journal on Software Tools for Technology Transfer 21(3), 307-324 (Jun 2019). https://doi.org/10.1007/s10009-019-00508-4

10. Boker, U.: Why These Automata Types? In: EPiC Series in Computing. vol. 57, pp. 143-163. EasyChair (Oct 2018). https://doi.org/10.29007/c3bj

11. Chatterjee, K., Gaiser, A., Křetínský, J.: Automata with Generalized Rabin Pairs for Probabilistic Model Checking and LTL Synthesis. In: Sharygina, N., Veith, H. (eds.) Computer Aided Verification. pp. 559-575. Lecture Notes in Computer Science, Springer, Berlin, Heidelberg (2013). https://doi.org/10.1007/978-3-642-39799-8_37

12. Courcoubetis, C., Yannakakis, M.: The complexity of probabilistic verification. Journal of the ACM 42(4), 857-907 (Jul 1995). https://doi.org/10.1145/210332.210339

13. Couvreur, J.M.: On-the-fly Verification of Linear Temporal Logic. In: Wing, J.M., Woodcock, J., Davies, J. (eds.) FM'99 — Formal Methods. pp. 253271. Lecture Notes in Computer Science, Springer, Berlin, Heidelberg (1999). https://doi.org/10.1007/3-540-48119-2_16

14. Duret-Lutz, A.: Contributions to LTL and $\omega$-Automata for Model Checking. Habilitation thesis, Université Pierre et Marie Curie (2017)

15. Duret-Lutz, A., Lewkowicz, A., Fauchille, A., Michaud, T., Renault, É., Xu, L.: Spot 2.0 - A Framework for LTL and $\omega$-Automata Manipulation. In: Artho, C., Legay, A., Peled, D. (eds.) Automated Technology for Verification and Analysis. pp. 
122-129. Lecture Notes in Computer Science, Springer International Publishing, Cham (2016). https://doi.org/10.1007/978-3-319-46520-3_8

16. Duret-Lutz, A., Poitrenaud, D., Couvreur, J.M.: On-the-fly Emptiness Check of Transition-Based Streett Automata. In: Automated Technology for Verification and Analysis (ATVA). LNCS, Springer (2009)

17. Esparza, J., Křetínský, J., Sickert, S.: One Theorem to Rule Them All: A Unified Translation of LTL into $\omega$-Automata. In: Proceedings of the 33rd Annual ACM/IEEE Symposium on Logic in Computer Science. pp. 384-393. LICS '18, Association for Computing Machinery, New York, NY, USA (Jul 2018). https://doi.org/10.1145/3209108.3209161

18. Giannakopoulou, D., Lerda, F.: From States to Transitions: Improving Translation of LTL Formulae to Büchi Automata. In: Peled, D.A., Vardi, M.Y. (eds.) Formal Techniques for Networked and Distributed Sytems - FORTE 2002. pp. 308326. Lecture Notes in Computer Science, Springer, Berlin, Heidelberg (2002). https://doi.org/10.1007/3-540-36135-9_20

19. Hahn, E.M., Li, G., Schewe, S., Turrini, A., Zhang, L.: Lazy Probabilistic Model Checking without Determinisation. arXiv:1311.2928 [cs] (Apr 2015), http://arxiv org/abs/1311.2928

20. Hahn, E.M., Perez, M., Schewe, S., Somenzi, F., Trivedi, A., Wojtczak, D.: Goodfor-MDPs Automata for Probabilistic Analysis and Reinforcement Learning. In: Biere, A., Parker, D. (eds.) Tools and Algorithms for the Construction and Analysis of Systems. pp. 306-323. Lecture Notes in Computer Science, Springer International Publishing, Cham (2020). https://doi.org/10.1007/978-3-030-45190-5_17

21. John, T., Jantsch, S., Baier, C., Klüppelholz, S.: Determinization and Limitdeterminization of Emerson-Lei Automata - Supplementary material (ATVA'21) (2021). https://doi.org/10.6084/m9.figshare.14838654.v2

22. Klein, J., Müller, D., Baier, C., Klüppelholz, S.: Are Good-for-Games Automata Good for Probabilistic Model Checking? In: Dediu, A.H., Martín-Vide, C., SierraRodríguez, J.L., Truthe, B. (eds.) Language and Automata Theory and Applications. pp. 453-465. Lecture Notes in Computer Science, Springer International Publishing, Cham (2014). https://doi.org/10.1007/978-3-319-04921-2_37

23. Křetínský, J., Meggendorfer, T., Sickert, S.: Owl: A Library for $\$ \$ \backslash$ omega $\$ \$$-Words, Automata, and LTL. In: Lahiri, S.K., Wang, C. (eds.) Automated Technology for Verification and Analysis. pp. 543-550. Lecture Notes in Computer Science, Springer International Publishing, Cham (2018). https://doi.org/10.1007/978-3-030-010904_34

24. Kwiatkowska, M., Norman, G., Parker, D.: PRISM 4.0: Verification of Probabilistic Real-Time Systems. In: Gopalakrishnan, G., Qadeer, S. (eds.) Computer Aided Verification. pp. 585-591. Lecture Notes in Computer Science, Springer, Berlin, Heidelberg (2011). https://doi.org/10.1007/978-3-642-22110-1_47

25. Löding, C., Pirogov, A.: Determinization of Büchi Automata: Unifying the Approaches of Safra and Muller-Schupp. In: Baier, C., Chatzigiannakis, I., Flocchini, P., Leonardi, S. (eds.) 46th International Colloquium on Automata, Languages, and Programming (ICALP 2019). Leibniz International Proceedings in Informatics (LIPIcs), vol. 132, pp. 120:1-120:13. Schloss Dagstuhl-Leibniz-Zentrum fuer Informatik, Dagstuhl, Germany (2019). https://doi.org/10.4230/LIPIcs.ICALP.2019.120

26. Major, J., Blahoudek, F., Strejček, J., Sasaráková, M., Zbončáková, T.: ltl3tela: LTL to Small Deterministic or Nondeterministic Emerson-Lei Automata. In: Automated Technology for Verification and Analysis (ATVA) (2019) 
27. Miyano, S., Hayashi, T.: Alternating finite automata on $\omega$-words. Theoretical Computer Science 32(3), 321-330 (Jan 1984). https://doi.org/10.1016/03043975(84)90049-5

28. Müller, D.: Alternative Automata-based Approaches to Probabilistic Model Checking. Ph.D. thesis, Technische Universität Dresden (Nov 2019), https://tud.qucosa.de/landing-page/?tx_dlf [id] =https $\% 3 \mathrm{~A} \% 2 \mathrm{~F} \% 2 \mathrm{Ftud}$. qucosa. de $\% 2$ Fapi $\% 2$ Fqucosa $\% 253 \mathrm{~A} 36100 \% 2 \mathrm{Fmets}$

29. Müller, D., Sickert, S.: LTL to Deterministic Emerson-Lei Automata. In: Games, Automata, Logics and Formal Verification (GandALF). EPTCS (2017)

30. Muller, D.E., Schupp, P.E.: Simulating alternating tree automata by nondeterministic automata: New results and new proofs of the theorems of Rabin, McNaughton and Safra. Theoretical Computer Science 141(1), 69-107 (Apr 1995). https://doi.org/10.1016/0304-3975(94)00214-4

31. Pnueli, A., Rosner, R.: On the synthesis of a reactive module. In: Proceedings of the 16th ACM SIGPLAN-SIGACT Symposium on Principles of Programming Languages. pp. 179-190. POPL '89, Association for Computing Machinery, New York, NY, USA (Jan 1989). https://doi.org/10.1145/75277.75293

32. Redziejowski, R.R.: An Improved Construction of Deterministic Omega-automaton Using Derivatives. Fundamenta Informaticae 119(3-4), 393-406 (Jan 2012). https://doi.org/10.3233/FI-2012-744

33. Renkin, F., Duret-Lutz, A., Pommellet, A.: Practical "Paritizing" of EmersonLei Automata. In: Hung, D.V., Sokolsky, O. (eds.) Automated Technology for Verification and Analysis. pp. 127-143. Lecture Notes in Computer Science, Springer International Publishing, Cham (2020). https://doi.org/10.1007/978-3-030-591526 67

34. Safra, S., Vardi, M.Y.: On omega-automata and temporal logic. In: Proceedings of the Twenty-First Annual ACM Symposium on Theory of Computing. pp. 127-137. STOC '89, Association for Computing Machinery, New York, NY, USA (Feb 1989). https://doi.org/10.1145/73007.73019

35. Safra, S.: Complexity of Automata on Infinite Objects. Ph.D. thesis, Weizmann Institute of Science, Rehovot, Israel (1989)

36. Schewe, S., Varghese, T.: Tight Bounds for the Determinisation and Complementation of Generalised Büchi Automata. In: Chakraborty, S., Mukund, M. (eds.) Automated Technology for Verification and Analysis. pp. 42-56. Lecture Notes in Computer Science, Springer, Berlin, Heidelberg (2012). https://doi.org/10.1007/9783-642-33386-6_5

37. Sickert, S., Esparza, J., Jaax, S., Křetínský, J.: Limit-Deterministic Büchi Automata for Linear Temporal Logic. In: Chaudhuri, S., Farzan, A. (eds.) Computer Aided Verification. pp. 312-332. Lecture Notes in Computer Science, Springer International Publishing, Cham (2016). https://doi.org/10.1007/978-3-319-41540-6_17

38. Vardi, M.Y.: Automatic verification of probabilistic concurrent finite state programs. In: 26th Annual Symposium on Foundations of Computer Science (Sfcs 1985). pp. 327-338 (Oct 1985). https://doi.org/10.1109/SFCS.1985.12

\section{A Proofs for Sec. 3}

Lemma 3.1. It holds that $\mathcal{L}(\mathcal{A})=\bigcup_{1 \leq i \leq m} \mathcal{L}(\operatorname{split}(\mathcal{A})[i])$. 
Proof. "ᄃ": Let $\rho$ be a run of $\mathcal{A}$ for $w$ such that $\rho=\alpha$. Then, it follows that $\rho \models \alpha_{i}$ for some $1 \leq i \leq m$. But then $\rho$ is also an accepting run of $\operatorname{split}(\mathcal{A})[i]$ and hence $w \in \mathcal{L}(\operatorname{split}(\mathcal{A})[i])$.

"卫": Let $\rho$ be an accepting run of $\operatorname{split}(\mathcal{A})[i]$ for some $1 \leq i \leq m$. Then $\rho \models \alpha_{i}$, and hence $\rho \models \alpha$. It follows that $\rho$ is an accepting run of $\mathcal{A}$.

$\alpha=\operatorname{Fin}(1) \wedge \operatorname{Inf}(1)$

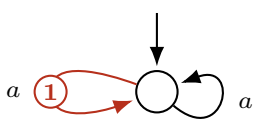

(a) $\mathcal{A}$

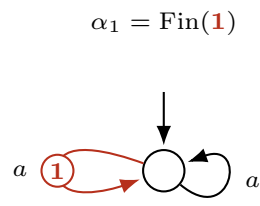

(b) $\mathcal{A}_{1}$

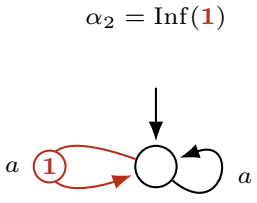

(c) $\mathcal{A}_{2}$

Fig. 5: An example showing that the analogue of Lem. 3.1 for conjunction and intersection is not correct. In other words, the intersection of languages of automata one gets by splitting along a top-level conjunction is not necessarily the language of the original automaton. In this example we have: $\mathcal{L}(\mathcal{A})=\varnothing$ and $\mathcal{L}\left(\mathcal{A}_{1}\right)=\mathcal{L}\left(\mathcal{A}_{2}\right)=\left\{a^{\omega}\right\}$. But then $\mathcal{L}(\mathcal{A}) \neq \mathcal{L}\left(\mathcal{A}_{1}\right) \cap \mathcal{L}\left(\mathcal{A}_{2}\right)$.

Proposition A.1. Let $\mathcal{A}_{i}=\left(Q_{i}, \Sigma, \delta_{i}, I_{i}, \alpha_{i}\right)$, with $i \in\{0,1\}$, be two complete TELA with disjoint state-spaces. The following statements hold:

1. $\mathcal{L}\left(\mathcal{A}_{0}\right) \cup \mathcal{L}\left(\mathcal{A}_{1}\right)=\mathcal{L}\left(\mathcal{A}_{0} \oplus \mathcal{A}_{1}\right)$

2. Assume that $\alpha_{i}=\operatorname{Inf}\left(T_{1}^{i}\right) \wedge \ldots \wedge \operatorname{Inf}\left(T_{k}^{i}\right)$, for $i \in\{0,1\}$. Then we have: $\mathcal{L}\left(\mathcal{A}_{0}\right) \cup \mathcal{L}\left(\mathcal{A}_{1}\right)=\mathcal{L}\left(\mathcal{A}_{0} \oplus_{G B A} \mathcal{A}_{1}\right)$

3. $\mathcal{L}\left(\mathcal{A}_{0}\right) \cup \mathcal{L}\left(\mathcal{A}_{1}\right)=\mathcal{L}\left(\mathcal{A}_{0} \otimes \mathcal{A}_{1}\right)$

Proof. 1.) This is clear as any accepting run of $\mathcal{A}_{i}$ (for $i \in\{0,1\}$ ) can be mapped directly to an accepting run of $\mathcal{A}_{0} \oplus \mathcal{A}_{1}$, and an accepting run $\mathcal{A}_{0} \oplus \mathcal{A}_{1}$ corresponds to an accepting run of one of the automata $\mathcal{A}_{0}, \mathcal{A}_{1}$.

2.) " $\subseteq$ ": Let $\rho$ be an accepting run of $\mathcal{A}_{0}$ (w.l.o.g). Then $\inf (\rho) \cap T_{j}^{0} \neq \varnothing$ holds for all $1 \leq j \leq k$, and hence also $\inf (\rho) \cap\left(T_{j}^{0} \cup T_{j}^{1}\right) \neq \varnothing$. But then $\rho$ is also an accepting run of $\mathcal{A}_{0} \oplus_{G B A} \mathcal{A}_{1}$.

" $\supseteq$ ": Let $\rho$ be an accepting run of $\mathcal{A}_{0} \oplus_{G B A} \mathcal{A}_{1}$. Then $\inf (\rho) \cap\left(T_{j}^{0} \cup T_{j}^{1}\right)$ holds for all $1 \leq j \leq k$. Recall that $\delta_{0} \cap \delta_{1}=\varnothing$. We have either $\inf (\rho) \subseteq \delta_{0}$ or $\inf (\rho) \subseteq \delta_{1}$ and $T_{j}^{i} \subseteq \delta_{i}$ for all $1 \leq j \leq k$ and $i \in\{0,1\}$. As a consequence, there exists $i \in\{0,1\}$ such that $\inf (\rho) \cap T_{j}^{i}$ for all $1 \leq j \leq k$. But then $\rho$ is an accepting run of $\mathcal{A}_{i}$.

3.) " $\subseteq$ ": Let $\rho=q_{0} q_{1} \ldots$ be an accepting run of $\mathcal{A}_{0}$ (w.l.o.g.) for $w$. As $\mathcal{A}_{1}$ is complete, we find a run $\rho^{*}$ of $\mathcal{A}_{0} \otimes \mathcal{A}_{1}$ for $w$ such that $\rho^{*}=\left(q_{0}, q_{0}^{\prime}\right)\left(q_{1}, q_{1}^{\prime}\right) \ldots$, where $q_{0}^{\prime} q_{1}^{\prime} \ldots$ is a run of $\mathcal{A}_{1}$ for $w$. As $\rho \models \alpha_{0}$, it follows that $\rho^{*} \models \uparrow\left(\alpha_{0}\right)$ and hence $\rho^{*}$ is an accepting run of $\mathcal{A}_{0} \otimes \mathcal{A}_{1}$. 


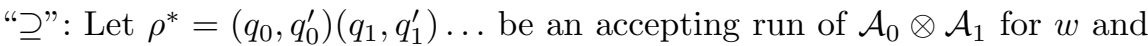
assume, w.l.o.g., that $\rho^{*} \models \uparrow\left(\alpha_{0}\right)$. Then $q_{0} q_{1}$, as a run for $w$, models $\alpha_{0}$ and hence it is an accepting run of $\mathcal{A}_{0}$.

$$
\alpha_{0}=\operatorname{Fin}(0)
$$

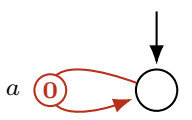

(a) $\mathcal{A}_{0}$

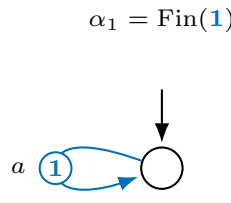

(b) $\mathcal{A}_{1}$

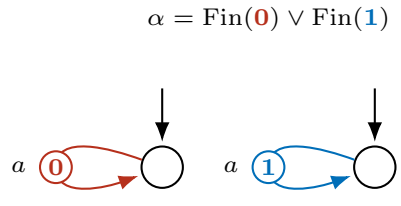

(c)

Fig. 6: An example showing why we need to add the conditions $\operatorname{Inf}\left(\delta_{0}\right)$ and $\operatorname{Inf}\left(\delta_{1}\right)$ when constructing the acceptance condition of $\mathcal{A}_{0} \oplus \mathcal{A}_{1}$. The TELA $\mathcal{A}_{0}$ and $\mathcal{A}_{1}$ both accept the empty language. If we unify the state spaces and disjunct the acceptance conditions we get the TELA in Figure 6c. However, this automaton accepts the language $a^{\omega} \neq \mathcal{L}\left(\mathcal{A}_{0}\right) \cup \mathcal{L}\left(\mathcal{A}_{1}\right)$.

Lemma 3.5. It holds that $\mathcal{L}(\mathcal{A})=\mathcal{L}(\operatorname{removeFin}(\mathcal{A}))=\mathcal{L}\left(\operatorname{removeFin}_{\mathrm{GBA}}(\mathcal{A})\right)$.

Proof. We first prove that $\mathcal{L}(\mathcal{A})=\mathcal{L}(\operatorname{removeFin}(\mathcal{A}))$.

" $\subseteq$ ": Let $\rho$ be an accepting run of $\mathcal{A}$ for $u$, and assume that $\rho \models \operatorname{Fin}\left(T_{0}^{i}\right) \wedge$ $\bigwedge_{1 \leq j \leq k_{i}} \operatorname{Inf}\left(T_{j}^{i}\right)$ (the $i$ 'th disjunct of acceptance condition $\alpha$, which is in DNF). It follows that there exists a position $K$ after which $\rho$ sees no transitions in $T_{0}^{i}$. We construct an accepting run $\rho^{\prime}$ of $\operatorname{removeFin}(\mathcal{A})$ for $u$ : for the first $K$ positions, it copies $\rho$ in the main copy $Q$. Then, it moves to $Q_{i}$, and continues to simulate the moves of $\rho$. From that fact that $\rho$ sees infinitely many transitions in each set $T_{j}^{i}$, it follows that $\rho^{\prime}$ models $\phi_{i}$ and hence is accepting.

" $\supseteq$ ": Let $\rho^{\prime}$ be an accepting run of $\operatorname{removeFin}(\mathcal{A})$ for $u$, i.e. $\rho^{\prime}=\phi_{i}$ for some $1 \leq i \leq m$. It follows that $\rho^{\prime}$ eventually moves to $Q_{i}$, because $\phi_{i}$ contains at least one Inf-atom whose all transitions are fully included in $Q_{i}$. As no other copy is reachable from $Q_{i}, \rho^{\prime}$ stays in $Q_{i}$ therafter. Recall that copy $Q_{i}$ does not contain any transition in $U_{0}^{i}$. Projecting the part of $\rho^{\prime}$ which is in $Q_{i}$ onto the corresponding transitions in $Q$ yields a run $\rho$ for $u$ in $\mathcal{A}$. From the definition of $\phi_{i}$ and the fact that $\rho^{\prime}$ models $\phi_{i}$ it follows directly that $\rho$ models $\alpha_{i}$, and hence $\rho$ is accepting.

We now show that $\mathcal{L}(\operatorname{removeFin}(\mathcal{A}))=\mathcal{L}\left(\operatorname{removeFin}_{\mathrm{GBA}}(\mathcal{A})\right)$ by proving that for all runs $\rho$ in $\left(\delta^{\prime}\right)^{\omega}$, the following equivalence holds: $\rho \models \alpha^{\prime} \Longleftrightarrow$ $\rho \models \alpha^{\prime \prime}$. We recall that $\alpha^{\prime}=\bigvee_{1 \leq i \leq m} \phi_{i}$, whith $\phi_{i}=\bigwedge_{1 \leq j \leq k_{i}} \operatorname{Inf}\left(U_{j}^{i}\right)$ and $\alpha^{\prime \prime}=\bigwedge_{1 \leq j \leq k} \operatorname{Inf}\left(U_{j}^{1} \cup \ldots \cup U_{j}^{m}\right)$, with $k=\max _{i} k_{i}$ and $U_{j}^{i}=\delta_{i}$ if $k_{i}<j \leq k$.

First, assume that $\rho \models \alpha^{\prime}$, which implies that there exists an $1 \leq i \leq m$ such that $\rho=\operatorname{Inf}\left(U_{j}^{i}\right)$ for all $1 \leq j \leq k_{i}$. It follows that $\inf (\rho) \subseteq \delta_{i}$ and hence also $\rho \models U_{j}^{i}$ for all $k_{i}<j \leq k$. But then clearly $\rho \models \operatorname{Inf}\left(U_{j}^{1} \cup \ldots \cup U_{j}^{m}\right)$ for all $1 \leq j \leq k$ and hence $\rho \models \alpha^{\prime \prime}$. 
Now assume that $\rho \models \alpha^{\prime \prime}$. Then, in particular, $\rho \models \operatorname{Inf}\left(U_{1}^{1} \cup \ldots \cup U_{1}^{m}\right)$, which implies that $\rho \models \operatorname{Inf}\left(U_{1}^{i}\right)$ for some $1 \leq i \leq m$. By construction of removeFin $(\mathcal{A})$ it follows that $\inf (\rho) \subseteq \delta_{i}$ and $\inf (\rho) \cap \delta_{i^{\prime}}=\varnothing$, for $i^{\prime} \neq i$. It follows that $\inf (\rho) \cap U_{j}^{i^{\prime}}=\varnothing$ for all $1 \leq j \leq k$ and $i^{\prime} \neq i$. The only possibility for $\rho$ to satisfy all conjuncts in $\alpha^{\prime \prime}$ is to satisfy $\inf (\rho) \cap \operatorname{Inf}\left(U_{j}^{i}\right) \neq \varnothing$ for all $1 \leq j \leq k$. But then it follows that $\rho \models \phi_{i}$ and hence $\rho \models \alpha^{\prime}$.

\section{B Proofs for Sec. 5}

We first show that there is a "canonical" partition $Q_{N}, Q_{D}$ for limit-determinism, which will be used in Props. 5.2 and 5.9 . Let $\mathcal{A}=(Q, I, \Sigma, \delta, \alpha)$ be a fixed TELA. We say that a state $q \in Q$ is deterministic if it has at most one successor in $\delta$ for each symbol, and define:

$$
Q_{D}^{*}=\left\{q \in Q \mid \text { all states } q^{\prime} \text { reachable from } q \text { are deterministic }\right\}
$$

and $Q_{N}^{*}=Q \backslash Q_{D}^{*}$. This partition can be computed in polynomial time using an $\mathrm{SCC}$ analysis of $\mathcal{A}$.

Lemma B.1. $\mathcal{A}$ is limit-deterministic iff the partition $Q_{D}^{*}, Q_{N}^{*}$ satisfies conditions 1-3 of Def. 5.1.

Proof. The direction from right to left is immediate. We now show that if there exists a partition $Q_{D}, Q_{N}$ which satisfies $1-3$, then so does $Q_{D}^{*}, Q_{N}^{*}$. By construction, $Q_{D}^{*}, Q_{N}^{*}$ satisfy conditions 1 . and 2. As every state in $Q_{D}$ is only allowed to reach deterministic states, it follows that $Q_{D} \subseteq Q_{D}^{*}$ and hence $Q_{N}^{*} \subseteq Q_{N}$. But then it follows from the fact that every accepting run of $\mathcal{A}$ eventually leaves $Q_{N}$ forever, that it also eventually leaves $Q_{N}^{*}$ forever. This shows that the partition $Q_{D}^{*}, Q_{N}^{*}$ satisfies condition 3 .

Proposition 5.2. Checking limit-determinism for TELA is coNP-complete.

Proof. As we have seen above, checking whether $\mathcal{A}$ is limit-deterministic amounts to checking whether the partition $Q_{D}^{*}, Q_{N}^{*}$ satisfies conditions 1-3 of Def. 5.1 Conditions 1-2 hold by construction. To check condition 3 one can construct an automaton $\mathcal{N}$ which contains all states and transitions of $Q_{N}$ and replaces all transitions out of $Q_{N}$ by a transition to a rejecting trap state (this may need a simple rewrite of the acceptance condition). Then, condition 3 holds if and only if $\mathcal{N}$ accepts the empty language, which can be checked in coNP for TELA (this follows from NP-completeness of the non-emptiness problem [1, Thm. 4.7]).

For coNP-hardness, we reduce from the emptiness problem of TELA, which is coNP-hard. First observe that an automaton $\mathcal{A}$ in which $Q_{D}^{*}$ is empty is limit-deterministic if and only if $\mathcal{L}(\mathcal{A})=\varnothing$. So it suffices to translate an arbitrary TELA $\mathcal{B}$ into a TELA $\mathcal{A}$ in which $Q_{D}^{*}$ is empty, and such that $\mathcal{L}(\mathcal{B})=\varnothing$ iff $\mathcal{L}(\mathcal{A})=\varnothing$. To do this we add a nondeterministic SCC to $\mathcal{B}$, a transition from each original state in $\mathcal{B}$ to that SCC, and make sure using the acceptance condition that no run which gets trapped in the new SCC is accepting. 
Proposition 5.4. $\bigoplus_{1 \leq i<m} \operatorname{det}\left(\operatorname{removeFin}\left(\mathcal{A}_{i}\right)\right)$ is limit-deterministic and of size $\sum_{1 \leq i \leq m}\left|\operatorname{det}\left(\mathcal{A}_{i}\right)\right|=m \cdot 2^{O(n(\log n+\log k))}$, where $k=\max \left\{k_{i} \mid 1 \leq i \leq m\right\}$.

Proof. The choice of initial component is the only nondeterminism of the resulting automaton. Hence we can take $Q_{D}$ to be the entire state-set and $Q_{N}=\varnothing$.

Proposition 5.9. Deciding $\operatorname{Pr}_{\mathcal{M}}^{\max }(\mathcal{L}(\mathcal{A}))>0$, given an $M D P \mathcal{M}$ and a limitdeterministic TELA $\mathcal{A}$, is NP-complete. If $\mathcal{A}$ has a fin-less acceptance condition, then the problem is in $P$.

Proof. NP-hardness of the problem for general limit-det of deterministic TELA follows directly from NP-hardness for deterministic TELA (see [28, Thm. 5.13]). For the upper-bounds we use the fact discussed above that if $\mathcal{A}$ is limit-deterministic, then we can compute a partition $Q_{D}, Q_{N}$ satisfying conditions 1-3 of Def. 5.1 in polynomial time.

In NP for limit-det. TELA. We now show that the problem of computing $\operatorname{Pr}_{\mathcal{M}}^{\max }(\mathcal{L}(\mathcal{A}))>0$ is in NP if $\mathcal{A}$ is limit-deterministic. Let $Q_{N}, Q_{D}$ be a partition satisfying conditions 1-3 of Def. 5.1 and let $\mathcal{M} \times \mathcal{A}$ be the product-MDP defined in Def. 5.10. Recall that an end-component of an MDP is a non-empty subset of its states $S^{\prime}$ together with a subset of the enabled actions $T(s) \subseteq \operatorname{Act}(s)$ for each state $s \in S^{\prime}$ such that the underlying graph is strongly connected and closed under probabilistic transitions [4, Definition 10.117]. An end-component $\mathcal{E}$ of $\mathcal{M} \times \mathcal{A}$ naturally induces a set of transitions of $\mathcal{A}$, which we denote by $\operatorname{atrans}(\mathcal{E})$.

Claim. $\operatorname{Pr}_{\mathcal{M}}^{\max }(\mathcal{L}(\mathcal{A}))>0$ holds iff there exists a reachable end-component $\mathcal{E}$ of $\mathcal{M} \times \mathcal{A}$ such that atrans $(\mathcal{E}) \models \alpha$.

"£": Let $(s, q)$ be a state contained in such an end-component $\mathcal{E}$. As $\mathcal{E}$ is assumed to be reachable, there exists a finite path $\pi=s_{0} \alpha_{0} s_{1} \alpha_{1} \ldots s_{n} \alpha_{n} s$ through $\mathcal{M}$ and a corresponding path $q_{0} \stackrel{L\left(s_{0} \ldots s_{n}\right)}{\longrightarrow} q$ through $\mathcal{A}$. We start defining a scheduler $\mathfrak{S}_{1}$ on $\mathcal{M}$ such that it chooses action $\alpha_{i}$ for all prefixes of length $i$, if $0 \leq i \leq n$ and $\alpha_{i}$ is enabled. Clearly, $\mathfrak{S}_{1}$ achieves a positive probability to realize the prefix $\pi$. As $\mathcal{E}$ is an end-component, we can construct a scheduler $\mathfrak{S}$ on $M \times \mathcal{A}$ from state $(s, q)$ such that the set of transitions visited infinitely often is the set of all transitions of $\mathcal{E}$, with probability one 4 , Lemma 10.119]. $\mathfrak{S}$ induces a scheduler $\mathfrak{S}_{2}$ on $\mathcal{M}$ from $s$ which satisfies: $\operatorname{Pr}_{\mathcal{M}, s}^{\mathfrak{S}_{2}}(\{\pi \mid L(\pi)$ is accepted from $q$ in $\mathcal{A}\})=1$. Combining schedulers $\mathfrak{S}_{1}$ and $\mathfrak{S}_{2}$ yields a scheduler witnessing $\operatorname{Pr}_{\mathcal{M}}^{\max }(\mathcal{L}(\mathcal{A}))>0$

" $\Longrightarrow$ ": Every path $\pi$ from $s$ in $\mathcal{M}$ induces a unique path $p_{q}(\pi)$ from $(s, q)$ in $\mathcal{M} \times \mathcal{A}$, if $q \in Q_{D}$ (assuming that $\mathcal{A}$ is complete). We let $\operatorname{Limit}(\pi)$ be the pair $(A, T)$ where $A$ is the set of states appearing infinitely often in path $\pi$ and $T: A \rightarrow 2^{\text {Act }}$ is the set of actions appearing infinitely often for each of the states in $A$. Given a state $q \in Q_{D}$ and an end-component $\mathcal{E}$ of $\mathcal{M} \times \mathcal{A}$, we let

$$
\begin{aligned}
X_{q, \mathcal{E}}=\left\{\pi \mid \text { there exist } \pi_{1} \pi_{2} \text { s.t. } \pi=\pi_{1} \pi_{2},\right. & \\
& \left.I \stackrel{L\left(\pi_{1}\right)}{\longrightarrow} \mathcal{A} q \text { and } \operatorname{Limit}\left(p_{q}\left(\pi_{2}\right)\right)=\mathcal{E}\right\}
\end{aligned}
$$

Let $\mathfrak{S}$ be a scheduler on $\mathcal{M}$ satisfying $\operatorname{Pr}_{\mathcal{M}}^{\mathfrak{S}}(\mathcal{L}(\mathcal{A}))>0$. The $\mathfrak{S}$-paths $\pi$ in $\mathcal{M}$ satisfying both $L(\pi) \in \mathcal{L}(\mathcal{A})$ and $\pi \notin \bigcup_{q \in Q_{D}}\left\{X_{q, \mathcal{E}} \mid \operatorname{atrans}(\mathcal{E}) \models \alpha\right\}$ form a 
null-set. This is because a path $\pi$ satisfies the following property with probability one under $\mathfrak{S}$ : for all $i \geq 0$ and $q \in Q_{D}$ : $\operatorname{Limit}\left(p_{q}(\pi[i .]).\right)$ forms an end-component. It follows that there exists $q \in Q_{D}$ and an end-component $\mathcal{E}$ of $\mathcal{M} \times \mathcal{A}$ satisfying atrans $(\mathcal{E}) \models \alpha$ such that $\operatorname{Pr}_{\mathcal{M}}^{\mathfrak{S}}\left(X_{q, \mathcal{E}}\right)>0$. But then $\mathcal{E}$ must also be reachable in $\mathcal{M} \times \mathcal{A}$, which concludes the proof of the claim.

It is a direct consequence now that the problem is in NP, as we can guess the end-component $\mathcal{E}$ and then check in polynomial time whether $\operatorname{atrans}(\mathcal{E}) \models \alpha$ holds.

In $\mathbf{P}$ for fin-less limit-det. TELA. If $\alpha$ is fin-less, then the existence of and end-component $\mathcal{E}$ satisfying $\operatorname{atrans}(\mathcal{E}) \models \alpha$ is equivalent to the existence of a maximal end-component satisfying the same property. This is because fin-less properties are preserved when adding additional transitions. As all maximal end-components can be enumerated in polynomial time (see Algorithm 47 in [4]), it follows by the above claim that $\operatorname{Pr}_{\mathcal{M}}^{\max }(\mathcal{L}(\mathcal{A}))>0$ can be decided in polynomial time in this case.

Before giving a proof of Thm. 5.7 we prove a few lemmas related to the breakpoint construction. Let $\mathcal{A}=\left(Q, q_{0}, \Sigma, \delta, \alpha\right)$ be a TELA in DNF, with $\alpha=\bigvee_{1 \leq i \leq m} \alpha_{i}$ and $\alpha_{i}=\operatorname{Fin}\left(T_{0}^{i}\right) \wedge \bigwedge_{1 \leq j \leq k_{i}} \operatorname{Inf}\left(T_{j}^{i}\right)$ (see Def. 3.3 and let the breakpoint automata $\mathcal{B P} \mathcal{P}_{i}$ be defined as in Def. 5.5. As defined in Sec. 5, we let $\theta$ be the extended subset transition function corresponding to $\delta$. Also, $\theta_{i}=\left.\theta\right|_{\delta \backslash T_{i}^{0}}$ is defined as $\theta$ but with $\delta$ restricted to transitions outside of $T_{i}^{0}$. We call a path a $\theta_{i}$-path if for all its transitions $\left(p, a, p^{\prime}\right)$ we have $p^{\prime} \in \theta_{i}(\{p\}, a)$. For an infinite word $w=w_{0} w_{1} w_{2} \ldots$ we let $w[j . . m]=w_{j} w_{j+1} \ldots w_{m}$.

The proofs follow known arguments for the correctness of limit-determinization for Büchi and generalized Büchi automata (see [12, Section 4.2] and 6, Sections 7.4 and 7.6]). Still, the extension to Emerson Lei requires additional arguments and hence we give the proofs here in our notation for completeness. The first lemma extends [6, Lemma 7.1].

Lemma B.2. For every accepting run $\rho=q_{0} q_{1} \ldots$ of $\mathcal{A}$ for $w=w_{0} w_{1} \ldots$ there exists an $1 \leq i \leq m$ such that $\rho \models \alpha_{i}$, and $a K \geq 0$ such that:

- for all $l \geq K:\left(q_{l}, w_{l}, q_{l+1}\right) \notin T_{0}^{i}$,

- for all $l \geq K$ there exists $m>l$ such that $\theta_{i}\left(\left\{q_{l}\right\}, w[l . . m]\right)=\theta_{i}\left(\left\{q_{K}\right\}, w[K . . m]\right)$.

Proof. As $\rho$ is accepting there must exist an $1 \leq i \leq m$ such that $\rho \models \alpha_{i}$, which implies $\rho \models \operatorname{Fin}\left(T_{0}^{i}\right)$. Fix such an $i$. Then, the existence of a $K$ (let us call it $K_{1}$ ) satisfying the first condition follows directly. Fix such a $K_{1}$.

Suppose, for contradiction, that for all $K_{2} \geq K_{1}$ there exists $l_{1} \geq K_{2}$ such that for all $m>l_{1}$ we have $\theta_{i}\left(\left\{q_{l_{1}}\right\}, w\left[l_{1} . . m\right]\right) \neq \theta_{i}\left(\left\{q_{K_{2}}\right\}, w\left[K_{2} . . m\right]\right)$. Clearly then $\theta_{i}\left(\left\{q_{l_{1}}\right\}, w\left[l_{1} . . m\right]\right) \subset \theta_{i}\left(\left\{q_{K_{2}}\right\}, w\left[K_{2} . . m\right]\right)$ as $q_{l_{1}} \in \theta_{i}\left(\left\{q_{K_{2}}\right\}, w\left[K_{2} . . l_{1}-1\right]\right)$. Applying the same argument lets us find $l_{2} \geq l_{1}$ such that for all $m>l_{2}$ we have $\theta_{i}\left(\left\{q_{l_{2}}\right\}, w\left[l_{2} . . m\right]\right) \subset \theta_{i}\left(\left\{q_{l_{1}}\right\}, w\left[l_{1} . . m\right]\right)$. Iterating this argument lets us construct an infinitely descending chain, which is impossible as all considered sets are finite.

It follows that there exists a $K_{2} \geq K_{1}$ satisfying the second property, which concludes the proof. 
Lemma B.3. $\mathcal{L}\left(\mathcal{G}_{\mathcal{A}}^{\mathrm{GFM}}\right) \subseteq \mathcal{L}(\mathcal{A})$ and $\mathcal{L}\left(\mathcal{G}_{\mathcal{A}}^{\mathrm{LD}}\right) \subseteq \mathcal{L}(\mathcal{A})$.

Proof. We give the argument only for $\mathcal{G}_{\mathcal{A}}^{\mathrm{GFM}}$, as it is the same for $\mathcal{G}_{\mathcal{A}}^{\mathrm{LD}}$ with simple modifications. Any accepting run of $\mathcal{G}_{\mathcal{A}}^{\mathrm{GFM}}$ for any $w_{0} w_{1} \ldots \in \Sigma^{\omega}$ has the form

$$
I \rightarrow Q_{1} \rightarrow \ldots \rightarrow Q_{k} \rightarrow\left(P_{0}, \varnothing, 0\right)_{i} \rightarrow\left(P_{1}, B_{1}, h_{1}\right)_{i} \rightarrow \ldots
$$

where the first part from $I$ to $Q_{k}$ is a path through the initial component of $\mathcal{G}_{\mathcal{A}}^{\mathrm{GFM}}$, and the subsequent part is a path through some breakpoint component $\mathcal{B} \mathcal{P}_{i}$, with $1 \leq i \leq m$. As $\mathcal{B} \mathcal{P}_{i}$ is the standard breakpoint automaton for $\mathcal{A}$ under acceptance $\alpha_{i}$ and after removing transitions in $T_{0}^{i}$, it follows from the soundness of the known construction [6, Lemma 7.3] that there exists a run $\rho$ through $\mathcal{A}$ from some state $q \in P_{0}$ for the word $w[k+1 .$.$] such that \rho=\alpha_{i}$ and $\rho$ sees no transition in $T_{0}^{i}$. Furthermore, clearly any state in $P_{0}$ is reachable from $I$ with a path labeled by $w[0 . . k]$. Concatenating such a path with the run $\rho$ yields an accepting run of $\mathcal{A}$ for $w_{0} w_{1} \ldots$

The last part of the following proof is essentially the proof of [6. Lemma 7.2].

Theorem 5.7. $\mathcal{G}_{\mathcal{A}}^{\mathrm{LD}}$ and $\mathcal{G}_{\mathcal{A}}^{\mathrm{GFM}}$ are syntactically limit-deterministic and satisfy $\mathcal{L}\left(\mathcal{G}_{\mathcal{A}}^{\mathrm{LD}}\right)=\mathcal{L}\left(\mathcal{G}_{\mathcal{A}}^{\mathrm{GFM}}\right)=\mathcal{L}(\mathcal{A})$. Their number of states is in $O\left(n+3^{n} m k\right)$ for $\mathcal{G}_{\mathcal{A}}^{\mathrm{LD}}$ and $O\left(2^{n}+3^{n} m k\right)=O\left(|\alpha|^{2} \cdot 3^{n}\right)$ for $\mathcal{G}_{\mathcal{A}}^{\mathrm{GFM}}$, where $k=\max \left\{k_{i} \mid 1 \leq i \leq m\right\}$.

Proof. As $\delta_{i}^{\text {break }} \subseteq \delta_{i}$ holds for all $i$, and the breakpoint components are deterministic, both $\mathcal{G}_{\mathcal{A}}^{\overline{\mathrm{GFM}}}$ and $\mathcal{G}_{\mathcal{A}}^{\mathrm{LD}}$ are syntactically limit-deterministic. The bound $O\left(2^{n}+3^{n} m k\right)$ on the states of $\mathcal{G}_{\mathcal{A}}^{\mathrm{GFM}}$ follows as the state-space of $\mathcal{B} \mathcal{P}_{i}$ is bounded by $3^{n} \cdot k$ (this uses that $B \subset R$ holds for states $(R, B, l)$ of $\mathcal{B} \mathcal{P}_{i}$, and $\left.l \leq k\right)$ for all $i$, and there are $m$ breakpoint components. Additionally, the initial component is a direct subset construction of $\mathcal{A}$, adding another $2^{n}$ states. For $\mathcal{G}_{\mathcal{A}}^{\mathrm{LD}}$, the initial component is of size $n$, hence it has state-complexity $O\left(n+3^{n} m k\right)$.

It remains to show that $\mathcal{L}\left(\mathcal{G}_{\mathcal{A}}^{\mathrm{LD}}\right)=\mathcal{L}\left(\mathcal{G}_{\mathcal{A}}^{\mathrm{GFM}}\right)=\mathcal{L}(\mathcal{A})$. The inclusions in $\mathcal{L}(\mathcal{A})$ follow by Lem. B.3 For the other direction, let $\rho=q_{0} q_{1} \ldots$ be an accepting run of $\mathcal{A}$ for $w$. By Lem. B.2 there exist $1 \leq i \leq m$ and $K \geq 0$ such that:

$-\rho \models \alpha_{i}$

- for all $l \geq K:\left(q_{l}, w_{l}, q_{l+1}\right) \notin T_{0}^{i}$,

- for all $l \geq K$ there exists $m>l$ such that $\theta_{i}\left(\left\{q_{l}\right\}, w[l . . m]\right)=\theta_{i}\left(\left\{q_{K}\right\}, w[K . . m]\right)$.

We define a run of $\mathcal{G}_{\mathcal{A}}^{\mathrm{GFM}}$ for $w$ as follows. For the first $K-1$ steps, it remains inside the initial component. It will reach a state $P \subseteq Q$ with $q_{K-1} \in P$. Then it takes the transition $\left(P, w[K-1],\left(\left\{q_{K}\right\}, \varnothing, 0\right)_{i}\right)$, and continues deterministically thereafter in component $\mathcal{B} \mathcal{P}_{i}$. This transition exists as $\left(q_{K-1}, w[K-1], q_{K}\right) \in \delta$, and hence $\left\{q_{K}\right\} \subseteq \theta(P, w[K-1])$. We argue that the remaining run $\left(\left\{q_{K}\right\}, \varnothing, 0\right)\left(R_{1}, B_{1}, h_{1}\right) \ldots$ sees infinitely many transitions in $\delta_{i}^{\text {break }}$. For contradiction, suppose that this is not the case. Then, for some $N \geq K$ we have: for all $j \geq N: B_{j} \subset R_{j}$ and $h_{j}=h_{j+1}$. Furthermore, there exists $N_{1}>N$ such that $\left(q_{N_{1}}, w\left[N_{1}\right], q_{N_{1}+1}\right) \in T_{i}^{h_{j}}$ as $\rho \models \alpha_{i}$. It follows that $q_{N_{1}+1} \in B_{N_{1}+1}$. By the third property above, however, there exists $m>N_{1}+1 \geq K$ such that $R_{m}=\theta_{i}\left(\left\{q_{K}\right\}, w[K . . m]\right)=$ $\theta_{i}\left(\left\{q_{N_{1}+1}\right\}, w\left[N_{1}+1 . . m\right]\right) \subseteq B_{m}$. This contradicts $B_{m} \subset R_{m}$. 
A run for $\mathcal{G}_{\mathcal{A}}^{\mathrm{LD}}$ can be constructed in the same way, with the only exception that the initial part of the run is set to be $q_{0} \ldots q_{K-1}$ (rather than the unique path in the subset-construction for prefix $w[0 . . K-2])$. In total, this shows $\mathcal{L}(\mathcal{A})=$ $\mathcal{L}\left(\mathcal{G}_{\mathcal{A}}^{\mathrm{GFM}}\right)$ and $\mathcal{L}(\mathcal{A})=\mathcal{L}\left(\mathcal{G}_{\mathcal{A}}^{\mathrm{LD}}\right)$, which concludes the proof.

Next, we turn to the proof that $\mathcal{G}_{\mathcal{A}}^{\mathrm{GFM}}$ is good-for-MDPs. First, we prove a lemma which gives a sufficient condition for a word to be accepted by $\mathcal{B P}$.

Lemma B.4. Let $1 \leq i \leq m, w \in \Sigma^{\omega}, 0=j_{0}<j_{1}<j_{2}<\ldots$ be an increasing sequence of natural numbers and $Q^{\prime} \subseteq Q$ satisfying:

- for all $l \geq 0: \theta_{i}\left(Q^{\prime}, w\left[j_{l} . . j_{l+1}-1\right]\right)=Q^{\prime}$ and

- for all $l \geq 0$ and $q^{\prime} \in Q^{\prime}$ there exists $q \in Q^{\prime}$ and a finite $\theta_{i}$-path from $q$ to $q^{\prime}$ in $\mathcal{A}$ labeled by $w\left[j_{l} . . j_{l+1}-1\right]$ which hits all transition-sets $T_{1}^{i}, \ldots, T_{k_{i}}^{i}$.

Then, $w$ is accepted from state $\left(Q^{\prime}, \varnothing, 0\right)$ in $\mathcal{B P}_{i}$.

Proof. As the first component of states in $\mathcal{B} \mathcal{P}_{i}$ just simulates the subset transition function $\theta_{i}$, the run from state $\left(Q^{\prime}, \varnothing, 0\right)$ of $\mathcal{B P}{ }_{i}$ for $w$ has the form:

$$
\left(Q^{\prime}, \varnothing, 0\right) \stackrel{w\left[0 . . j_{1}-1\right]}{\longrightarrow}\left(Q^{\prime}, B_{1}, h_{1}\right) \stackrel{w\left[j_{1} . . j_{2}-1\right]}{\longrightarrow}\left(Q^{\prime}, B_{2}, h_{2}\right) \stackrel{w\left[j_{2} . . j_{3}-1\right]}{\longrightarrow} \ldots
$$

We show that each infix $\left(Q^{\prime}, B_{l}, h_{l}\right) \stackrel{w\left[j_{l} . j_{l+1}-1\right]}{\longrightarrow}\left(Q^{\prime}, B_{l+1}, h_{l+1}\right)$ of the run above sees a transition in $\delta_{i}^{\text {break }}$. For contradiction, suppose that for some $l$ this is not the case. Then the third component is not changed in any transition of the corresponding infix run. Let

$$
\left(Q_{0}, A_{0}, h_{l}\right) \stackrel{w\left[j_{l}\right]}{\longrightarrow}\left(Q_{1}, A_{1}, h_{l}\right) \stackrel{w\left[j_{l}+1\right]}{\longrightarrow} \ldots \stackrel{w\left[j_{l}+M-1\right]}{\longrightarrow}\left(Q_{M}, A_{M}, h_{l}\right)
$$

be the sequence of the run between $\left(Q_{0}, A_{0}, h_{l}\right)=\left(Q^{\prime}, B_{l}, h_{l}\right)$ and $\left(Q_{M}, A_{M}, h_{l}\right)=$ $\left(Q^{\prime}, B_{l+1}, h_{l}\right)$. Take arbitrary $q^{\prime} \in Q^{\prime} \backslash B_{l+1}$ and let $P$ be the source states of the incoming $w\left[j_{l}+M-1\right]$-transitions of $q^{\prime}$ wrt. $\delta \backslash T_{0}^{i}$. It follows that $P \cap A_{M-1}$ is empty and for no $p \in Q_{M-1}$ we have $\left(p, w\left[j_{l}+M-1\right], q^{\prime}\right) \in T_{h_{l}}^{i}$, as otherwise we would either have $q^{\prime} \in B_{l+1}$ or see a break-point transition. In particular, there is no path from any $p \in Q_{M-1}$ to $q^{\prime}$ labeled by $w\left[j_{l}+M-1\right]$ that sees $T_{h_{l}}^{i}$. We can continue this argument inductively until reaching $\left(Q_{0}, A_{0}, h_{l}\right)$ and conclude that there is no $q \in Q^{\prime}$ such that there exists a $\theta_{i}$-path from $q$ to $q^{\prime}$ labeled by $w\left[j_{l} . . j_{l+1}-1\right]$ which sees some transition in $T_{h_{l}}^{i}$. This is in contradiction with the second property above.

Hence the run sees a transition in $\delta_{i}^{\text {break }}$ infinitely often, which implies that it is accepting.

Recall that removeFin $(\operatorname{split}(\mathcal{A})[i])$ is a GBA with two components: the initial component $Q_{1}$ is a copy of $\mathcal{A}$ where no transition is accepting, and component $Q_{2}$ is a copy of $\mathcal{A}$ without the transitions of $T_{0}^{i}$. The acceptance condition is the GBA condition $\alpha_{i}=\bigwedge_{1 \leq j \leq k_{i}} \operatorname{Inf}\left(U_{j}^{i}\right)$ where $U_{j}^{i}$ is the set of transitions corresponding to $T_{j}^{i} \backslash T_{0}^{i}$ in the component $Q_{2}$. 
Now let $\mathcal{D}_{i}=\operatorname{det}(\operatorname{removeFin}(\operatorname{split}(\mathcal{A})[i]))$ be the Rabin automaton one gets by applying the construction of $[36]$ to removeFin $(\operatorname{split}(\mathcal{A})[i])$. Their soundnessproof [36, Thm. 1] can be modified slightly to prove the following statement. Let $\gamma$ be the extended subset transition function of removeFin $(\operatorname{split}(\mathcal{A})[i])$.

Lemma B.5. Let $d_{0} d_{1} \ldots$ be an accepting run of $\mathcal{D}_{i}$ for $w$ satisfying Rabin pair $\operatorname{Inf}\left(D_{1}\right) \wedge \operatorname{Fin}\left(D_{2}\right)$ and $i_{0}$ be a position such that for all $l \geq i_{0}$ we have $\left(d_{l}, w[l], d_{l+1}\right) \notin D_{2}$ and for infinitely many $l \geq i_{0}$ we have $d_{l}=d_{i_{0}}$.

Then, there exists a sequence $i_{0}<i_{1}<i_{2} \ldots$ and $a Q^{\prime} \subseteq Q_{2}$ such that

$-Q^{\prime} \subseteq \gamma\left(I, w\left[0 . . i_{0}-1\right]\right)$,

- for all $l \geq 0: Q^{\prime}=\gamma\left(Q^{\prime}, w\left[i_{l} . . i_{l+1}-1\right]\right)$ and for all $q_{1} \in Q^{\prime}$ there exists a $q_{2} \in Q^{\prime}$ and a finite $\gamma$-path for $w\left[i_{l} . . i_{l+1}-1\right]$ hitting all transition-sets $U_{1}^{i}, \ldots, U_{k_{i}}^{i}$.

Proof. The following statement follows directly from the proof of [36, Thm. 1]. Let $j_{0}<j_{1}<\ldots$ be an infinite sequence of positions such that: for $l \geq j_{0}$ we have $\left(d_{l}, w[l], d_{l+1}\right) \notin D_{2}$ and the sequence $d_{j_{l}} w\left[j_{l}\right] d_{j_{l}+1} \ldots d_{j_{l+1}}$ sees $k_{i}$ transitions in $D_{1}$ for all $l \geq 0$. Then there exists a sequence $P_{0}, P_{1} \ldots$, with all $P_{l} \subseteq Q_{1} \cup Q_{2}$, such that $P_{0} \subseteq \gamma\left(I, w\left[0 . . j_{0}-1\right]\right), P_{l+1}=\gamma\left(P_{l}, w\left[j_{l} . . j_{l+1}-1\right]\right)$ and for all $q_{1} \in P_{l+1}$ there exists a $\gamma$-path for $w\left[j_{l} . . j_{l+1}-1\right]$ hitting all transition sets $U_{1}^{i}, \ldots, U_{k_{i}}^{i}$. Furthermore, the sequence is such that if $d_{j_{l}}=d_{j_{p}}$, then $P_{l}=P_{p}$.

Clearly a sequence $j_{0}<j_{1}<\ldots$ exists satisfying the above assumption (as $\rho$ is accepting) and which additionally has the property that $d_{j_{l}}=d_{j_{0}}$ for all $l \geq 0$ (by taking $j_{0}=i_{0}$ and the fact that infinitely many positions see $d_{i_{0}}$ ). We may conclude that the sequence $P_{0}, P_{1} \ldots$ exists as above and as $d_{j_{l}}=d_{j_{0}}$ for all $l \geq 0$, indeed $P_{0}=P_{l}$ for all $l$. So we may take $Q^{\prime}=P_{0}$, and the only thing left to show is that $Q^{\prime} \subseteq Q_{2}$ holds. This, however, follows from the fact that $Q^{\prime}$ is reachable from some accepting transition.

We are now in a position to prove Lem. 5.11. Recall that $\mathcal{D}=\bigotimes_{1 \leq i \leq m} \mathcal{D}_{i}$ and $\mathfrak{S}$ is a finite memory scheduler on $\mathcal{M}$.

Lemma 5.11. Let $\mathfrak{s}$ be a state in an accepting $B S C C B$ of $\mathcal{M}_{\mathfrak{S}} \times \mathcal{D}$ and $\pi_{1}$ be a finite path that reaches $\mathfrak{s}$ from the initial state of $\mathcal{M}_{\mathfrak{S}} \times \mathcal{D}$. Then, there exists $1 \leq i \leq m$ and $Q^{\prime} \subseteq \theta\left(I, L\left(\pi_{1}\right)\right)$ such that:

$$
\operatorname{Pr}_{\mathfrak{s}}\left(\left\{\pi \mid L(\pi) \text { is accepted from }\left(Q^{\prime}, \varnothing, 0\right) \text { in } \mathcal{B} \mathcal{P}_{i}\right\}\right)=1
$$

Proof. There exists some Rabin pair $\operatorname{Inf}\left(D_{1}^{\prime}\right) \wedge \operatorname{Fin}\left(D_{2}^{\prime}\right)$ of $\mathcal{D}$ such that no transition of $B$ is contained in $D_{2}^{\prime}$, and some transition of $B$ is contained in $D_{1}^{\prime}$. By construction of $\mathcal{D}$, this pair directly corresponds to a $\operatorname{Rabin}$ pair $\operatorname{Inf}\left(D_{1}\right) \wedge \operatorname{Fin}\left(D_{2}\right)$ of one of the components $\mathcal{D}_{i}$ (as the acceptance condition of $\mathcal{D}$ is essentially the disjunction of the acceptance conditions of the $\mathcal{D}_{i}$ ).

Hence for every path $\pi_{2}$ through $B$ starting in $\mathfrak{s}$ which sees all transitions in $B$ infinitely often we find an accepting run $d_{0} d_{1} \ldots$ of $\mathcal{D}_{i}$ for $L\left(\pi_{1} \pi_{2}\right)$ and a sequence $j_{0}<j_{1}<\ldots$ where $j_{0}=\left|\pi_{1}\right|$ and such that:

- no transition of $d_{j_{0}} d_{j_{0}+1} \ldots$ is included in $D_{2}$, 
- for all $l \geq 0: d_{j_{l}}=d_{j_{0}}$ and the run $d_{0} d_{1} \ldots$ sees an accepting transition in between position $j_{l}$ and $j_{l+1}$ for all $l \geq 0$.

By Lem. B.5 there exists a $Q^{\prime} \subseteq \gamma\left(I, L\left(\pi_{1}\right)\right) \cap Q_{2}$ such that for all $l \geq 0$ : $Q^{\prime}=\gamma\left(Q^{\prime}, w\left[j_{l} . . j_{l+1}-1\right]\right)$ and for all $q_{1} \in Q^{\prime}$ there exists a $q_{2} \in Q^{\prime}$ and a finite $\gamma$-path for $w\left[j_{l} . . j_{l+1}-1\right]$ hitting all transition-sets $U_{1}^{i}, \ldots, U_{k_{i}}^{i}$. Let $Q^{\prime \prime}$ be the corresponding set of states of $\mathcal{A}$, which implies $Q^{\prime \prime} \subseteq \theta\left(I, L\left(\pi_{1}\right)\right)$. As $Q^{\prime} \subseteq Q_{2}$ we have: for all $q_{1} \in Q^{\prime \prime}$ there exists a $q_{2} \in Q^{\prime \prime}$ and a finite $\theta_{i}$-path for $w\left[j_{l} . . j_{l+1}-1\right]$ hitting all transition-sets $T_{1}^{i}, \ldots, T_{k_{i}}^{i}$.

It follows that the conditions of Lem. B.4 are satisfied and hence that $L\left(\pi_{2}\right)$ is accepted from $\left(Q^{\prime \prime}, \varnothing, 0\right)$ in $\mathcal{B} \mathcal{P}_{i}$.

Lemma 5.13. For every finite-memory scheduler $\mathfrak{S}$ on $\mathcal{M}$, there exists a scheduler $\mathfrak{S}^{\prime}$ on $\mathcal{M} \times \mathcal{G}_{\mathcal{A}}^{\mathrm{GFM}}$ such that:

$$
\operatorname{Pr}_{\mathcal{M} \times \mathcal{G}_{\mathcal{A}}^{\mathrm{GFM}}}^{\mathfrak{S}^{\prime}}\left(\Pi_{a c c}\right) \geq \operatorname{Pr}_{\mathcal{M}}^{\mathfrak{S}}(\mathcal{L}(\mathcal{A}))
$$

Proof. Let $\mathfrak{S}$ be a finite-memory scheduler on $\mathcal{M}$ and $\mathcal{M}_{\mathfrak{S}} \times \mathcal{D}$ be as above. We construct the scheduler $\mathfrak{S}^{\prime}$ as follows. For every finite path $\pi_{1}$ of $\mathcal{M} \times \mathcal{G}_{\mathcal{A}}^{\mathrm{GFM}}$ it needs to choose an action of $\mathcal{M}$ and whether to move from the initial component to one of the breakpoint components (this is the only nondeterministic choice in $\mathcal{G}_{\mathcal{A}}^{\mathrm{GFM}}$ ). The action of $\mathcal{M}$ is always chosen in the same way as by $\mathfrak{S}$ for the corresponding finite path of $\mathcal{M}$. If the path in $\mathcal{M}_{\mathfrak{S}} \times \mathcal{D}$ corresponding to $\pi_{1}$ does not end in a BSCC, $\mathfrak{S}^{\prime}$ chooses to remain in the initial component. If it does reach a BSCC $B$ we make a distinction on whether $B$ is accepting or not. If $B$ is not accepting, then $\mathfrak{S}^{\prime}$ can be defined arbitrarily for all prefixes that extend $\pi_{1}$. If $B$ is accepting, then let us assume that last $\left(\pi_{1}\right)=(s, P)$ is the current state of $\mathcal{M} \times \mathcal{G}_{\mathcal{A}}^{\mathrm{GFM}}$. Then $P$ is exactly the set of states reachable in $\mathcal{A}$ from $I$ on a path labeled by $L^{\times}\left(\pi_{1}\right)$. By Lem. 5.11 there exists $1 \leq i \leq k$ and $Q^{\prime} \subseteq P$ such that the probability of generating a suffix $\pi_{2}$ from state $s$ in $\mathcal{M}$ under scheduler $\mathfrak{S}$ whose label is accepted in $\mathcal{B P}_{i}$ from state $\left(Q^{\prime}, \varnothing, 0\right)$ is 1 . Consequently, $\mathfrak{S}^{\prime}$ chooses $\left(\theta\left(Q^{\prime}, L(s)\right), \varnothing, 0\right)_{i}$ as successor state of the automaton and continues to simulate $\mathfrak{S}$. As the probability of generating a path $\pi$ such that $L(\pi) \in \mathcal{L}(\mathcal{A})$ in $\mathcal{M}_{\mathfrak{S}}$ is equivalent to the probability of reaching an accepting BSCC in $\mathcal{M}_{\mathfrak{S}} \times \mathcal{D}$, we can conclude that $\operatorname{Pr}_{\mathcal{M} \times \mathcal{G}_{\mathcal{A}}^{\mathrm{GFM}}}^{\mathfrak{G}^{\prime}}\left(\Pi_{a c c}\right) \geq \operatorname{Pr}_{\mathcal{M}}^{\mathfrak{G}}(\mathcal{L}(\mathcal{A}))$.

Proposition 5.14. The automaton $\mathcal{G}_{\mathcal{A}}^{\mathrm{GFM}}$ is good-for-MDP.

Proof. To show that $\mathcal{G}_{\mathcal{A}}^{\mathrm{GFM}}$ is GFM, it suffices to show the following two statements:

1. For every finite memory scheduler $\mathfrak{S}$ of $\mathcal{M}$ there exists a scheduler $\mathfrak{S}^{\prime}$ of $\mathcal{M} \times \mathcal{G}_{\mathcal{A}}^{\mathrm{GFM}}$ such that

$$
\operatorname{Pr}_{\mathcal{M}}^{\mathfrak{S}}(\mathcal{L}(\mathcal{A})) \leq \operatorname{Pr}_{\mathcal{M} \times \mathcal{G}_{\mathcal{A}}^{\mathrm{GFM}}}^{\mathfrak{S}^{\prime}}\left(\Pi_{a c c}\right)
$$

2. For every finite memory scheduler $\mathfrak{S}$ of $\mathcal{M} \times \mathcal{G}_{\mathcal{A}}^{\mathrm{GFM}}$ there exists a scheduler $\mathfrak{S}^{\prime}$ of $\mathcal{M}$ such that

$$
\operatorname{Pr}_{\mathcal{M} \times \mathcal{G}_{\mathcal{A}}^{\mathrm{GFM}}}^{\mathfrak{G}}\left(\Pi_{a c c}\right) \leq \operatorname{Pr}_{\mathcal{M}}^{\mathfrak{S}^{\prime}}(\mathcal{L}(\mathcal{A}))
$$


1. is proved by Lem. 5.13 and 2 . is proved for arbitrary automaton in 22 , Thm. $1]$.

\section{Additional evaluation of experiments}

Fig. 7 compares the approaches pairwise on the random benchmark and on the $D N F$ benchmark. On the random benchmark, the comparisons of product and good-for-MDP with SPOT show that the results are widely spread, i.e. there is no clear correlation. Both approaches produce in many cases smaller automata than Sрот. Most automata produced by limit-det. are smaller than the results produced by Sрот. Such behavior is expected as the complexity is only singleexponential instead of double-exponential. The approach limit-det. via GBA produces in almost all cases smaller results than the approach limit-det. On the $D N F$ benchmark product produces in most cases smaller results than Sрот.

Tab. 2 compares the approaches Spot and product in more detail. The input automata are grouped by the amount of nondeterminism and the length of the input acceptance condition, which is in DNF. These groups are the same as in Fig. 4. The values "states", "time" and "acceptance" are the median value of the ratio of these metrics, which are the values depicted in Fig. 4. If only one of the approaches was able to construct a result within the given time and memory bounds we define the ratio as 0 (SPOT did not construct a result) or infinity (product did not construct a result). If none of the approaches was able to construct a result, we do not consider this input automaton for the calculation. The number of timeouts and memouts increases with the amount of nondeterminism and the size of the input acceptance condition. product produces for all subsets automata with fewer states than SPOT and the ratio of the computation time decreases the larger the amount of nondeterminism is. On the $D N F$ benchmark, product produces significantly smaller automata while the acceptance condition is only slightly larger.

Fig. 8 compares the approaches on the sequence of automata described in Fig. 2. For these automata, SPOT first computes GBA with an exponential number of acceptance sets. The example also highlights the effect of the langcover heuristic: it benefits from the fact that the language of the input automaton under any individual disjunct of the DNF is the same. This is used to prune large parts of the state-space. 

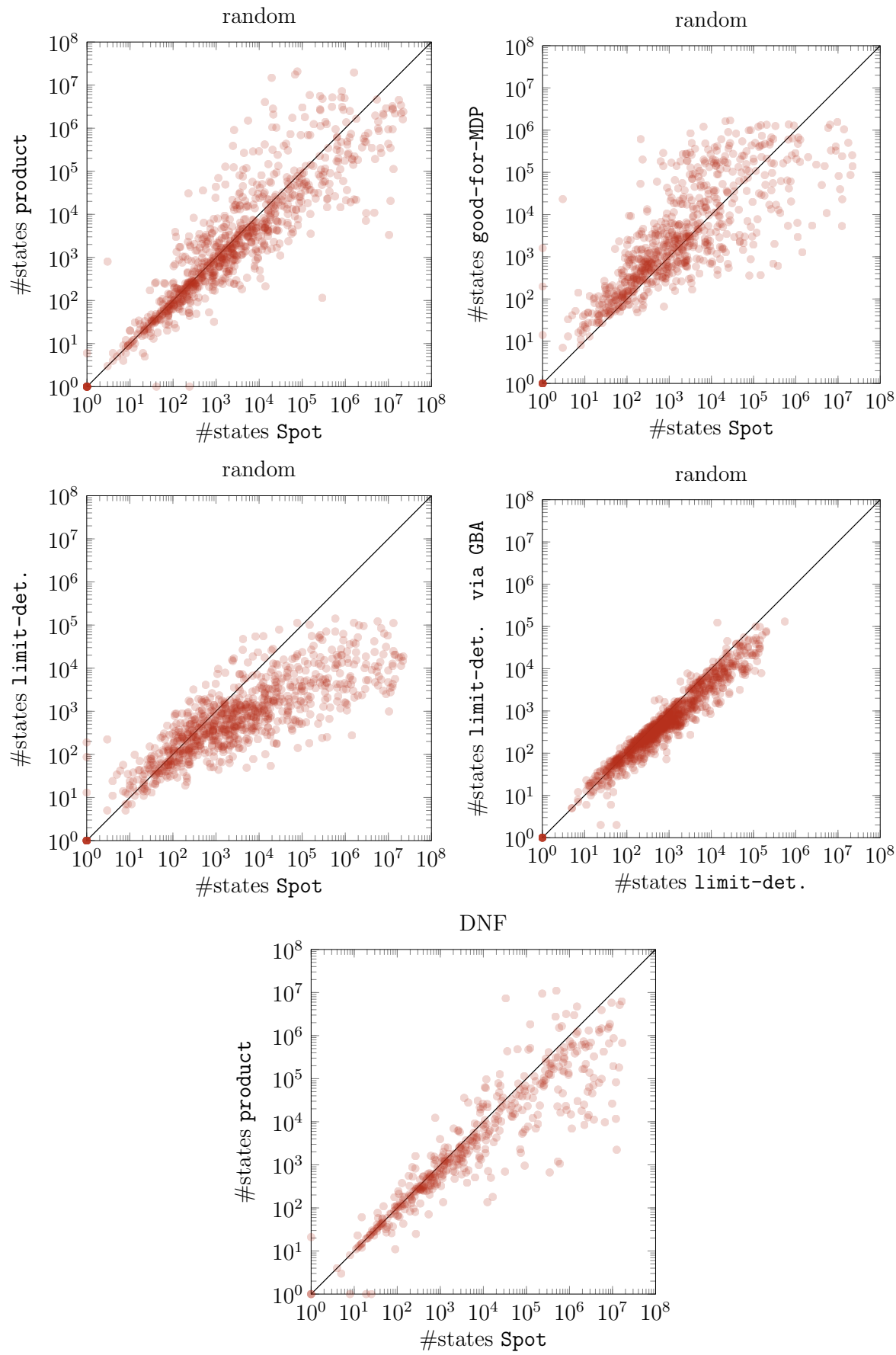

Fig. 7: Comparing pairwise the sizes of the state spaces for different approaches on the random benchmark and DNF benchmark. 
Table 2: Comparison of SPOT and product, with input automata grouped by the size of the DNF of their acceptance condition and the amount of nondeterminism. "states", "time" and "acceptance" refer to the median of the ratio product / Sрот. The number of automata that is denoted in brackets is the number of input automata for which both approaches were able to construct a result within the given time and memory bounds.

\begin{tabular}{|c|c|c|c|c|c|c|c|c|c|c|}
\hline \multirow{2}{*}{ benchmark } & \multirow{2}{*}{$\begin{array}{l}\text { amount of } \\
\text { nondet. }\end{array}$} & \multirow{2}{*}{$\begin{array}{l}\text { input } \\
\text { acceptance }\end{array}$} & \multirow{2}{*}{$\begin{array}{l}\text { \#automata } \\
\text { (no time-/memouts) }\end{array}$} & \multicolumn{2}{|c|}{ timeouts } & \multicolumn{2}{|c|}{ memouts } & \multirow{2}{*}{ states } & \multirow{2}{*}{ time } & \multirow{2}{*}{ acceptance } \\
\hline & & & & SPOT & product & SPOT & product & & & \\
\hline \multirow{6}{*}{ random } & \multirow{2}{*}{$\leq 0.66$} & $2 \leq|\alpha| \leq 11$ & $175(175)$ & $0.0 \%$ & $0.0 \%$ & $0.0 \%$ & $0.0 \%$ & 0.91 & 1.37 & 1.80 \\
\hline & & $12 \leq|\alpha| \leq 21$ & $180(180)$ & $0.0 \%$ & $0.0 \%$ & $0.0 \%$ & $0.0 \%$ & 0.82 & 1.44 & 1.64 \\
\hline & \multirow{2}{*}{$>0.66, \leq 1.33$} & $2 \leq|\alpha| \leq 11$ & $162(159)$ & $0.0 \%$ & $0.0 \%$ & $0.0 \%$ & $1.9 \%$ & 0.85 & 1.19 & 1.80 \\
\hline & & $12 \leq|\alpha| \leq 21$ & $159(144)$ & $0.0 \%$ & $0.6 \%$ & $3.1 \%$ & $7.5 \%$ & 0.75 & 1.26 & 1.82 \\
\hline & \multirow{2}{*}{$>1.33$} & $2 \leq|\alpha| \leq 11$ & $163(128)$ & $1.8 \%$ & $0.0 \%$ & $16.0 \%$ & $11.7 \%$ & 0.75 & 0.84 & 1.72 \\
\hline & & $12 \leq|\alpha| \leq 21$ & $161(82)$ & $1.2 \%$ & $7.5 \%$ & $42.2 \%$ & $28.0 \%$ & 0.43 & 0.80 & 1.67 \\
\hline \multirow{3}{*}{ DNF } & $\leq 0.66$ & "2 $2 \leq|\alpha| \leq 21$ & "193(193) & 些0.0\% & 年0\% & 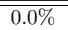 & $0.0 \%$ & $\overline{c 0.81}$ & 1.17 & 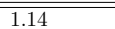 \\
\hline & $>0.66$, & $2 \leq|\alpha| \leq 21$ & $167(160)$ & $0.0 \%$ & $0.0 \%$ & $3.0 \%$ & $2.4 \%$ & 0.68 & 0.93 & 1.19 \\
\hline & $>1.33$ & $2 \leq|\alpha| \leq 21$ & $140(111)$ & $1.4 \%$ & $0.0 \%$ & $18.6 \%$ & $10.7 \%$ & 0.26 & 0.25 & 1.00 \\
\hline
\end{tabular}

comparison of sizes of the state spaces

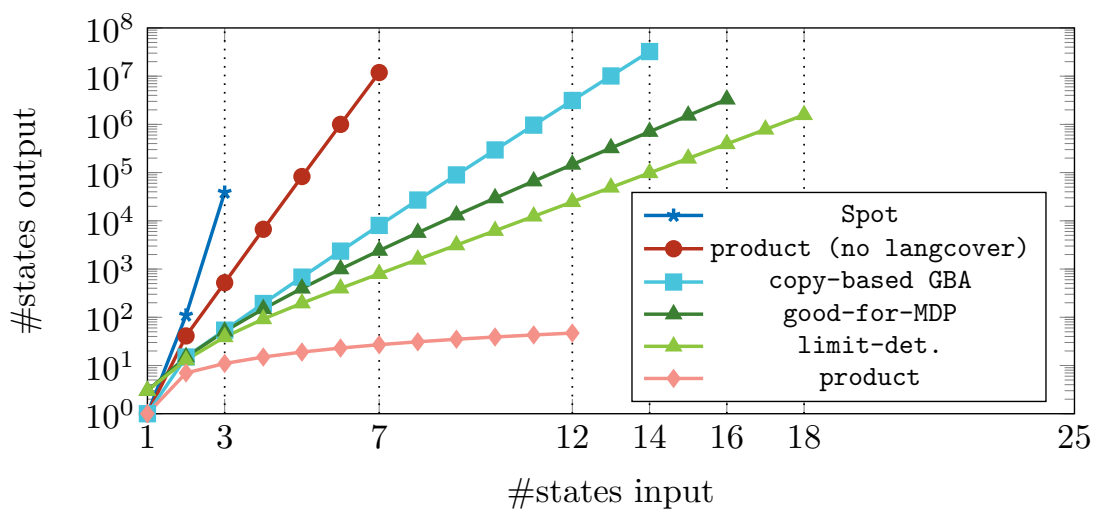

Fig. 8: Comparing the approaches for input automata of Fig. 2, The copy-based GBA approaches produce the same results and are collapsed here. 